\title{
REDSHIFT EVOLUTION IN BLACK HOLE-BULGE RELATIONS: TESTING C IV-BASED BLACK HOLE MASSES
}

\author{
Jenny E. Greene ${ }^{1,4}$, Chien Y. Peng ${ }^{2}$, and Randi R. Ludwig ${ }^{3}$ \\ ${ }^{1}$ Department of Astrophysical Sciences, Princeton University, Princeton, NJ 08544, USA \\ ${ }^{2}$ NRC Herzberg Institute of Astrophysics, 5071 West Saanich Road, Victoria, BC V9E2E7, Canada \\ ${ }^{3}$ University of Texas at Austin, Department of Astronomy, 1 University Station, C1400 Austin, TX 78712, USA \\ Received 2009 September 17; accepted 2009 December 9; published 2010 January 8
}

\begin{abstract}
We re-examine claims for redshift evolution in black hole-bulge scaling relations based on lensed quasars. In particular, we refine the black hole $(\mathrm{BH})$ mass estimates using measurements of Balmer lines from near-infrared spectroscopy obtained with Triplespec at Apache Point Observatory. In support of previous work, we find a large scatter between Balmer and UV line widths, both Mg II $\lambda \lambda 2796,2803$ and C IV $\lambda \lambda 1548,1550$. There is tentative evidence that C III] $\lambda 1909$, despite being a blend of multiple transitions, may correlate well with $\mathrm{Mg}$ II, although a larger sample is needed for a real calibration. Most importantly, we find no systematic changes in the estimated BH masses for the lensed sample based on Balmer lines, providing additional support to the interpretation that black holes were overly massive compared to their host galaxies at high redshift.
\end{abstract}

Key words: galaxies: active - galaxies: nuclei - galaxies: Seyfert

Online-only material: color figures

\section{EVOLUTION IN BLACK HOLE-BULGE RELATIONS}

Locally, we observe tight correlations between the properties of bulge-dominated galaxies and the masses of their central supermassive black holes (BHs; e.g., Kormendy \& Richstone 1995; Tremaine et al. 2002; Gültekin et al. 2009). The mechanisms that establish and maintain these relations are uncertain, despite innumerable suggestions in the literature (e.g., Silk \& Rees 1998; Murray et al. 2005; Miralda-Escudé \& Kollmeier 2005; Hopkins et al. 2006; Peng 2007). In principle, evaluating the demographics of nuclear $\mathrm{BHs}$ as a function of redshift should observationally constrain the processes that lead to the tight scaling relations observed today. Unfortunately, it is currently prohibitive to obtain dynamical $\mathrm{BH}$ masses for systems beyond tens of Mpc. Thus, estimates of BH mass at large distance are necessarily based on very indirect methods linked to accretion processes in active BHs (e.g., Vestergaard 2002).

Several studies have used active galaxies to probe evolution in BH-bulge scaling relations, probing redshifts from $0.4<$ $z<0.6$ (Woo et al. 2006; Treu et al. 2007; Woo et al. 2008) and $1 \lesssim z \lesssim 4$ (Peng et al. 2006a, 2006b; Salviander et al. 2007; Shields et al. 2006; Ho 2007; Jahnke et al. 2009; McLeod $\&$ Bechtold 2009) all the way to $z=6.4$ (Walter et al. 2004). Generally speaking, a wide variety of observations suggest that $\mathrm{BH}$-bulge relations do evolve with redshift, in the sense that the ratio of $\mathrm{BH}$ to bulge mass was higher at early times (but see also Shields et al. 2003; Alexander et al. 2008), although we have observational constraints only for the most massive systems $\left(M_{\mathrm{BH}}>10^{8} M_{\odot}\right)$ at high redshift. This counterintuitive result has stimulated vigorous discussion both about the ramifications for the co-evolution of BHs and bulges (e.g., Robertson et al. 2006; Croton 2006) and about whether there are built-in biases in the measurement techniques (Lauer et al. 2007).

Unfortunately, even apart from potential population biases, our interpretation of the observations is prone to significant uncertainty. On the one hand, it is only possible to obtain $\mathrm{BH}$

\footnotetext{
${ }^{4}$ Russell, Princeton-Carnegie Fellow.
}

mass estimates at cosmological distances using active galaxies (typically luminous quasars at high redshift). Immediately, it becomes very challenging to characterize the host galaxy properties, when the quasar outshines the underlying galaxy starlight by factors of $\sim 10-30$ (e.g., Peng et al. 2006a; Kim et al. 2008a). Apart from direct imaging, some groups have used gas measurements (predominantly $\mathrm{CO}$ ) to obtain dynamical masses (Shields et al. 2006; Ho et al. 2008; Walter et al. 2004; Riechers et al. 2008, 2009), which may or may not provide a reliable tracer of the galaxy mass (Ho 2007). The width of narrow emission lines, particularly [O III] $\lambda 5007$, has also been substituted for the galaxy velocity dispersion (e.g., Shields et al. 2003; Boroson 2003; Salviander et al. 2007; Gaskell 2009). While there is a strong correlation between stellar and gaseous velocity dispersion in low-luminosity sources (e.g., Heckman et al. 1981; Nelson \& Whittle 1996; Greene \& Ho 2005a; Ho 2009), there is good reason to suspect that it does not hold at high luminosity (e.g., Greene et al. 2009). Peng et al. (2006b, P06 hereafter) mitigated the host galaxy contrast problem by focusing on lensed quasars. The quasars are lensed differently from the underlying (resolved) host galaxies, reducing the contrast problem discussed above. For that reason, we focus on the lensed quasar sample in this paper.

Daunting as measuring high-redshift galaxy mass and stellar velocity dispersion may be, particularly in the presence of a luminous quasar, the $\mathrm{BH}$ mass measurements are equally problematic. The techniques are indirect and model dependent. Briefly, active galaxies contain dense gas orbiting at distances of light days to months from the central $\mathrm{BH}$ that gives rise to broad emission lines with widths of thousands of $\mathrm{km} \mathrm{s}^{-1}$. By combining a size scale with the line width of the emitting region, the broad-line region (BLR) gas can be used as a dynamical tracer of the BH mass (e.g., Dibai 1980). Direct size estimates are obtained by measuring the time lag between variability in the continuum and line emission (reverberation mapping; Blandford \& McKee 1982; Peterson et al. 2004). A tight, empirically determined correlation between BLR radii and the luminosity of the active galaxy (the radius-luminosity relation; 
Table 1

Sample and Observations

\begin{tabular}{|c|c|c|c|c|c|c|c|}
\hline $\begin{array}{c}\text { Galaxy } \\
\text { (1) }\end{array}$ & $\begin{array}{l}\text { R.A. } \\
\text { (2) }\end{array}$ & $\begin{array}{c}\text { Decl. } \\
\text { (3) }\end{array}$ & $\begin{array}{c}m_{H} \\
(4) \\
\end{array}$ & $\begin{array}{c}\text { Obs. Date } \\
(5)\end{array}$ & $\begin{array}{r}t_{\exp } \\
(6) \\
\end{array}$ & $\begin{array}{c}\mathrm{S} / \mathrm{N}_{\mathrm{H} \alpha} \\
(7)\end{array}$ & $\begin{array}{c}\mathrm{S} / \mathrm{N}_{\mathrm{H} \beta} \\
(8) \\
\end{array}$ \\
\hline PMNJ0134-0931 & $01: 34: 35.7$ & $-09: 31: 02$ & 15.3 & 2009 Jul 14 & 3240 & 295.8 & 13.7 \\
\hline Q0142-100 & $01: 45: 16.5$ & $-09: 45: 17$ & 17.6 & 2008 Oct 18 & 2880 & 63.3 & $\ldots$ \\
\hline SDSS0246-0825 & $02: 46: 34.1$ & $-08: 25: 36$ & 18.1 & 2008 Nov 11 & 4320 & 111.2 & 14.3 \\
\hline MG0414+0534 & $04: 14: 37.7$ & $+05: 34: 44$ & 15.9 & 2008 Oct 18 & 3600 & 140.1 & 15.5 \\
\hline HS0810+2554 & $08: 13: 31.3$ & $+25: 45: 03$ & 14.8 & 2008 Oct 18 & 1800 & 393.7 & 54.3 \\
\hline SBS0909+523 & 09:13:01.1 & $+52: 59: 28$ & 14.7 & 2008 Oct 18 & 2160 & 374.6 & 163.7 \\
\hline FBQ0951+2635 & $09: 51: 22.6$ & $+26: 35: 14$ & 17.0 & 2008 Nov 11 & 2160 & 45.0 & 19.2 \\
\hline Q0957+561 & 10:01:20.8 & $+55: 53: 49$ & 15.6 & 2008 Nov 17 & 2880 & 460.1 & 111.1 \\
\hline J1004+1229 & $10: 04: 24.9$ & $+12: 29: 22$ & 17.8 & 2008 Nov 17 & 5400 & 125.9 & 17.2 \\
\hline HE1104-1805 & $11: 06: 33.5$ & $-18: 21: 24$ & 15.9 & 2009 Mar 14 & 2880 & 182.9 & 10.1 \\
\hline PG1115+080 & $11: 18: 17.0$ & $+07: 45: 57$ & 15.7 & 2008 Mar 24 & 1440 & 152.8 & 31.8 \\
\hline B $1152+200$ & $11: 55: 18.3$ & $+19: 39: 42$ & 15.6 & 2009 Jun 5 & 3600 & 50.6 & 9.2 \\
\hline $\mathrm{H} 1413+117$ & $14: 15: 46.4$ & $+11: 29: 41$ & 15.8 & 2009 May 5 & 3600 & 137.2 & 18.8 \\
\hline B $1422+231$ & $14: 24: 38.1$ & $+22: 56: 00$ & 14.4 & 2009 May 5 & 1440 & $\ldots$ & 39.9 \\
\hline FBQ1633+3134 & $16: 33: 49.0$ & $+31: 34: 11$ & 15.8 & 2009 May 11 & 1440 & 104.0 & 13.2 \\
\hline $\mathrm{Q} 2237+030$ & $22: 40: 30.3$ & $+03: 21: 28$ & 15.0 & 2009 Jul 14 & 3240 & 128.8 & 27.9 \\
\hline
\end{tabular}

Notes. Column 1: name; Column 2: right ascension (hrs; J2000); Column 3: declination (deg; J2000); Column 4: observed H-band magnitude (mag); Column 5: date of observation; Column 6: total on-source exposure time (sec); Column 7: signal-to-noise ratio summed over the H $\alpha$ emission line, excluding any regions that were excised from the fit; Column 8: same as Column 7 for the $\mathrm{H} \beta$ line.

Kaspi et al. 2005; Bentz et al. 2006, 2009a) can be used to obtain approximate BLR radii for the quasar population in general (e.g., Vestergaard 2002). The current data support a slope of $R_{\mathrm{BLR}} \propto L^{0.5}$, as is expected if neither the density structure of the BLR nor the shape of the ionizing continuum depends on luminosity (e.g., Bentz et al. 2006). Unfortunately, the radiusluminosity relation has not been calibrated extensively for luminosities greater than $L_{5100 \AA} \approx 10^{46} \mathrm{erg} \mathrm{s}^{-1}$ (see Kaspi et al. 2007).

Because the kinematic structure and inclination of the BLR are unknown, the derived virial "masses" $\left(M_{\mathrm{BH}} \propto v^{2} R_{\mathrm{BLR}} / G\right)$ have no physically motivated normalization. Recent reverberation mapping experiments reveal signatures of inflow, rotation, and outflow in individual objects, but such two-dimensional maps remain scarce (Welsh \& Horne 1991; Bentz et al. 2009c; Denney et al. 2009b). Our current practice is to compare the virial masses with independent estimates of $\mathrm{BH}$ mass, typically using the $M_{\mathrm{BH}}-\sigma_{*}$ relation, to derive an average scale factor (e.g., Gebhardt et al. 2000; Ferrarese et al. 2001; Onken et al. 2004; Nelson et al. 2004; Greene \& Ho 2006; Shen et al. 2008a). The fact that any correlation is seen between virial masses and the mass inferred from the $M_{\mathrm{BH}}-\sigma_{*}$ relation is encouraging, but leaves much room for large systematic uncertainties (e.g., Krolik 2001; Collin et al. 2006).

At higher redshift, only rest-frame UV spectra are readily available for large samples of quasars. The scaling relations for the UV lines (e.g., Mg II $22800 \AA$; C IV $\lambda 1550 \AA$ ) include additional layers of uncertainty. Virtually no reverberation mapping has been performed with $\mathrm{Mg}$ II, and the scaling relations for this line are simply scaled to match $\mathrm{H} \beta$ (e.g., McLure \& Dunlop 2004; Onken \& Kollmeier 2008). In the case of $\mathrm{C}$ IV, reverberation mapping has been done (e.g., Peterson et al. 2005), but there are strong reasons to suspect that the C IV line width is not dominated by virial motions (e.g., Gaskell 1982; Baldwin et al. 1996; Richards et al. 2002a; Leighly \& Moore 2004; Baskin \& Laor 2005; Sulentic et al. 2007; Shen et al. 2008b), although debate continues on this point (e.g., Vestergaard \& Peterson 2006; Kelly \& Bechtold 2007; Gavignaud et al. 2008; Dietrich et al. 2009). For these reasons, virial masses based on Balmer lines (preferably $\mathrm{H} \alpha$; Greene \& Ho 2005b) have the most credibility, since these have been directly compared with alternate estimates of $M_{\mathrm{BH}}$. We focus specifically on obtaining Balmer-based virial masses for the high-redshift lensed quasar sample from P06. Our primary goal is to determine whether the masses presented in P06 are systematically biased by the use of UV line transitions. We follow P06 and assume a standard cosmology with $H_{0}=$ $100 h=70 \mathrm{~km} \mathrm{~s}^{-1} \mathrm{Mpc}^{-1}, \Omega_{\mathrm{m}}=0.30$, and $\Omega_{\Lambda}=0.70$.

\section{OBSERVATIONS AND DATA REDUCTION}

The data presented here were obtained over the course of a year using the newly commissioned near-infrared spectrograph Triplespec (Wilson et al. 2004) at Apache Point Observatory (Table 1). All objects were observed with a $1.1 \times 43^{\prime \prime}$ slit and Fowler sampling of 8 (the number of non-destructive readouts at the beginning and end of each exposure designed to minimize readnoise; Fowler \& Gatley 1990). Triplespec covers a nominal wavelength range of $0.95-2.46 \mu \mathrm{m}$ with $R=3500$. Observing conditions ranged from clear to partly cloudy, with typical seeing of $\theta \approx 1^{\prime \prime} .5$. In most cases, the slit was positioned at the parallactic angle in the middle of the observation, although in a couple of cases we positioned the slit to place two quasar images in the slit at once. The object was dithered along the slit every $180 \mathrm{~s}$ to improve sky subtraction. For each quasar, we observed a nearby A0V star $(10<H<6 \mathrm{mag})$ to serve as flux and telluric standard.

The data were reduced using custom software that is a modified version of Spextool and is described in detail in Vacca et al. (2003) and Cushing et al. (2004). Using domeflat and arc-line exposures, the code creates and applies a flatfield and wavelength solution. Bias and dark subtraction is accomplished through pair-wise differencing of images taken at two slit positions, which also removes air glow emission from the atmosphere, at least to zeroth order. Nonlinearity corrections are applied, and then each pair of spectra is traced and optimally extracted (Horne 1986), including background subtraction. Wavelength calibration is applied, and all the spectra of a given source are median-combined. Flux calibration 
Table 2

Spectral Measurements

\begin{tabular}{|c|c|c|c|c|c|c|c|c|c|c|}
\hline $\begin{array}{l}\text { Name } \\
(1)\end{array}$ & $\begin{array}{c}\left.\mathrm{EW}_{[\mathrm{O}} \mathrm{III}\right] \\
(2)\end{array}$ & $\begin{array}{c}\mathrm{EW}_{\mathrm{Fe} \text { II }} \\
(3)\end{array}$ & $\begin{array}{c}\mathrm{EW}_{\mathrm{H} \beta, \mathrm{n}} \\
(4)\end{array}$ & $\begin{array}{c}\mathrm{EW}_{\mathrm{H} \beta, \mathrm{t}} \\
(5)\end{array}$ & $\begin{array}{c}\mathrm{EW}_{\mathrm{H} \alpha, \mathrm{n}} \\
(6)\end{array}$ & $\begin{array}{c}\left.\mathrm{EW}_{[\mathrm{N}} \mathrm{II}\right] \\
(7)\end{array}$ & $\begin{array}{c}\mathrm{EW}_{\mathrm{H} \alpha, \mathrm{t}} \\
(8)\end{array}$ & $\begin{array}{c}\alpha_{\mathrm{opt}} \\
(9)\end{array}$ & $\begin{array}{l}\alpha_{\mathrm{UV}} \\
(10)\end{array}$ & $\begin{array}{l}\alpha_{\mathrm{P} 06} \\
(11) \\
\end{array}$ \\
\hline PMNJ0134-0931 & 12 & 45 & 2.1 & 68 & 70. & 9.6 & 491 & -0.4 & $\ldots$ & $\ldots$ \\
\hline Q0142-100 & $\ldots$ & 11 & $\ldots$ & 42 & $<0.8$ & $<0.5$ & 212 & -2.2 & -1.5 & -1.8 \\
\hline SDSS0246-0825 & 6 & 58 & 1.5 & 50 & $<0.6$ & 4.8 & 212 & -2.4 & $\ldots$ & . . \\
\hline MG0414+0534 & $\ldots$ & 163 & 0.7 & 123 & 3.6 & 1.3 & 340 & 0.10 & $\ldots$ & 1.0 \\
\hline HS0810+2554 & 10 & 76 & $<0.5$ & 86 & $<0.7$ & $<0.5$ & 377 & -1.3 & -1.8 & .. \\
\hline SBS0909+523 & 63 & 0 & 7.5 & 119 & $<1.2$ & 16. & 526 & -1.3 & -0.9 & -1.0 \\
\hline FBQ0951+2635 & 43 & 157 & $<1.2$ & 128 & $<2.9$ & 9.5 & 340 & -1.4 & -1.0 & -1.7 \\
\hline Q0957+561 & 14 & 25 & 1.0 & 43 & $<0.5$ & 3.4 & 158 & -1.5 & -1.8 & -1.8 \\
\hline $\mathrm{J} 1004+1229$ & $\ldots$ & 60 & 9.4 & 76 & $<1.1$ & 0.9 & 327 & -0.6 & $\ldots$ & -1.5 \\
\hline HE1104-1805 & 9 & 31 & 1.2 & 64 & 12. & 8.7 & 230 & -2.5 & $\ldots$ & -2.0 \\
\hline PG1115+080 & $\ldots$ & 48 & $\ldots$ & 50 & $<0.7$ & $<0.5$ & 289 & -1.8 & -1.8 & -2.0 \\
\hline B $1152+200$ & $\ldots$ & $\ldots$ & $\ldots$ & $\ldots$ & 37. & 15. & 744 & 4.10 & 0.1 & $\ldots$ \\
\hline H1413+117 & 39 & 20 & 10. & 75 & $<1.2$ & 40. & 314 & -1.7 & -0.3 & -0.9 \\
\hline B $1422+231$ & 26 & 0 & 9.6 & 60 & $\ldots$ & $\ldots$ & $\ldots$ & -1.5 & $\ldots$ & -1.4 \\
\hline FBQ1633+3134 & 29 & 28 & 6.4 & 62 & $<0.9$ & $<0.5$ & 325 & -1.1 & -2.1 & -1.8 \\
\hline $\mathrm{Q} 2237+030$ & 12 & 10 & 0.7 & 30 & 11. & 5.2 & 114 & -1.5 & $\ldots$ & $\ldots$ \\
\hline
\end{tabular}

Notes. Column 1: name; Column 2: equivalent width (EW; $\AA$ ) of the [O III $]$ 25007 line. Here and in the following entries the EWs are measured from the multi-Gaussian fits. Column 3: EW ( $\AA$ ) of the Fe II line measured between $4434 \AA$ and $4684 \AA$, following Boroson \& Green (1992). Column 4: EW ( $\AA$ ) of the narrow component of $\mathrm{H} \beta$. Column 5: EW ( $\AA$ ) of the total $\mathrm{H} \beta$ line. Column 6: EW ( $\AA$ ) of the narrow component of H $\alpha$. Column 7: EW ( $(\AA)$ of [N II] $\lambda 6584 \AA$. Column 8: EW ( $\AA$ ) of the total H $\alpha$ line. Column 9: slope of the rest-frame optical continuum measured from the Triplespec spectra, $f_{\lambda} \propto \lambda^{\alpha_{\mathrm{opt}}}$. Column 10: slope of the rest-frame UV continuum measured from the SDSS spectra. Column 11: slope measured by P06 from broadband photometry (see the text).

is accomplished using an A0V star observed at similar time and air mass. This same star is used to create a model of the telluric absorption by assuming that the A star has an intrinsic spectrum identical to that of Vega (see Vacca et al. 2003 for details). Prior to correction, small wavelength shifts between the A star and the program object are derived on an order-by-order basis using a cross-correlation technique. Finally, the orders are merged with small-scale factors applied to properly match the edges of each order, and cosmetic data clipping is done. The software also generates an error array. Heliocentric corrections are calculated using the IRAF task bcvcorr.

The resulting $\mathrm{S} / \mathrm{N}$ for each object across the $\mathrm{H} \alpha$ and $\mathrm{H} \beta$ lines are shown in Table 1. The relative flux calibration is reasonable and the resulting spectra have smooth, power-law continua. However, given the variable clouds that plagued many of our observations, we suspect that the overall flux calibration scale is not reliable. For instance, the flux scales for two observations of B1152+200 differ by a factor of 3. Our results are not impacted by these problems, however, since we measure intrinsic luminosities from broadband photometry combined with a lens model (P06 and see below).

\subsection{Rest-frame Ultraviolet Spectra from SDSS}

In addition to the rest-frame optical spectra obtained with Triplespec, we also utilize observed optical (rest-frame ultraviolet; UV) spectra from the Sloan Digital Sky Survey (SDSS; York et al. 2000; Abazajian et al. 2009). These targets were selected from the SDSS photometry as quasars (Richards et al. 2002b). Galaxies with SDSS observations have tabulated UV slopes in Table 2.

\section{CONTINUUM AND LINE MEASUREMENTS}

\subsection{The Fits}

Quasar continua are generally well fit by a power law. However, superimposed on this smooth continuum is a "pseudocontinuum" of broad Fe II multiplet emission that effectively litters the entire optical/UV region of the spectrum. Although much progress has been made deriving theoretical Fe II spectra (e.g., Verner et al. 2004), the prospect of fitting $>800$ transitions individually is a daunting task. Rather, it is common practice to derive an $\mathrm{Fe}$ II template from a high $\mathrm{S} / \mathrm{N}$ observation of an active galaxy whose broad emission lines are intrinsically narrow $\left(\lesssim 1000 \mathrm{~km} \mathrm{~s}^{-1}\right)$, typically $1 \mathrm{Zw}$ I. Here we use the optical template of Boroson \& Green (1992) and the UV template presented in Salviander et al. (2007) that was derived from that of Vestergaard \& Wilkes (2001), but has a theoretical template pasted in beneath the Mg II line from Sigut \& Pradhan (2003). Vestergaard \& Wilkes also provide a separate Fe III template at $\approx 1900 \AA$ that we utilize in attempting to fit the $\left.\mathrm{C}_{\mathrm{III}}\right] \lambda 1909 \AA$ line. In addition, we follow Dietrich et al. (2002) and model the "little blue bump" (Grandi 1982) as a combination of higher order Balmer emission lines (Storey \& Hummer 1995) and bound-free Balmer continuum emission with a temperature $T_{e}=15,000 \mathrm{~K}$, electron density $n_{e}=10^{8}-10^{10} \mathrm{~cm}^{-3}$, and optical depth $0.1 \leqslant \tau_{v} \leqslant 2$ (following Grandi 1982).

In practice, we fit the power-law continuum, Fe II emission, and Balmer continuum simultaneously, using emission-linefree windows (see Figure 1). The fits include a normalization and slope for the (single) power-law component, a width, shift, and amplitude for the Fe template, and a density and optical depth for the Balmer continuum. In the UV, we find the best results fixing the $\mathrm{Fe}$ II broadening to that of $\mathrm{H} \beta$, while in the optical we find negligible difference in allowing the broadening to be free. We subtract the continuum model and create a lineonly spectrum.

We fit the emission line spectra with multi-component Gaussians. These components are used only to create a high-fidelity noise-free match to the emission lines; we do not ascribe physical meaning to them. We first fit the $\mathrm{H} \beta+\left[\mathrm{O}_{\text {III }}\right] \lambda \lambda 4959,5007$ region. The narrow [O III] lines are fit with up to three Gaussians each, with the relative wavelengths tied to laboratory values and the line intensities constrained to have a flux ratio of 1:3 (Table 2; see details in Greene \& Ho 

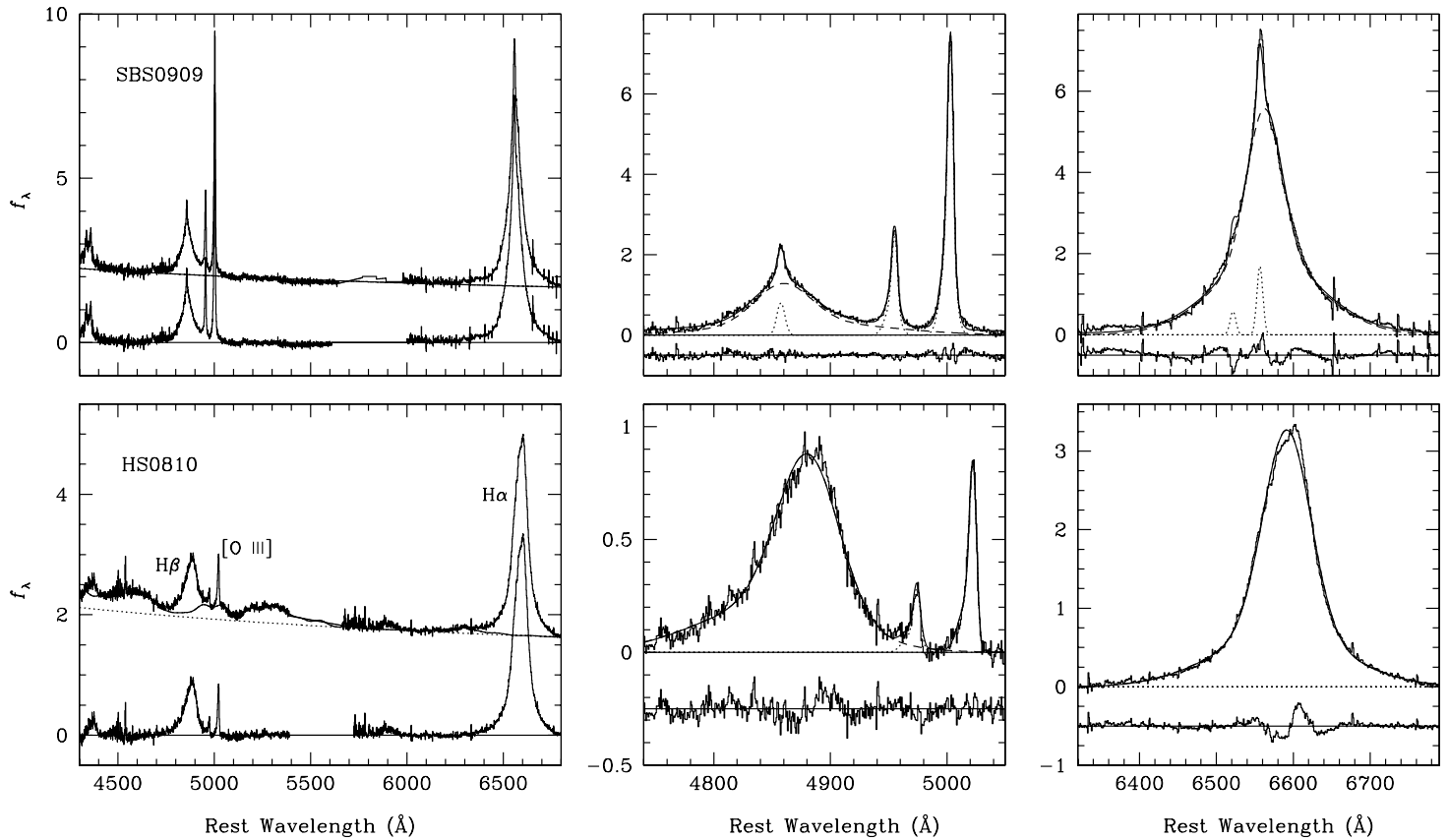

Figure 1. Example fits to the continuum (left), $\mathrm{H} \beta$ (middle), and $\mathrm{H} \alpha$ (right) lines from the Triplespec data. We show the data (solid histogram), the total model (thin solid), the broad- (dashed) and narrow-line (dotted) model components, and residuals below (thin solid histogram). Data are plotted with an arbitrary scale in $f_{\lambda}$. The rest of the sample are plotted in the appendix.

Table 3

Line-width Measurements

\begin{tabular}{|c|c|c|c|c|c|c|c|c|c|c|c|}
\hline $\begin{array}{l}\text { Name } \\
\text { (1) }\end{array}$ & $\begin{array}{c}z \\
(2)\end{array}$ & $\begin{array}{c}\left.\mathrm{FWHM}_{[\mathrm{O}} \mathrm{III}\right] \\
\text { (3) }\end{array}$ & $\begin{array}{c}\mathrm{FWHM}_{\mathrm{H} \alpha} \\
\text { (4) }\end{array}$ & $\begin{array}{c}\mathrm{FWHM}_{\mathrm{H} \beta} \\
(5)\end{array}$ & $\begin{array}{c}\mathrm{FWHM}_{\mathrm{Mg} \text { II }} \\
(6)\end{array}$ & $\begin{array}{c}\left.\mathrm{FWHM}_{\mathrm{C} I I]}\right] \\
\text { (7) }\end{array}$ & $\begin{array}{c}\mathrm{FWHM}_{\mathrm{CIV}} \\
(8)\end{array}$ & $\begin{array}{c}\lambda L_{5100 \AA} \\
\text { (9) }\end{array}$ & $\begin{array}{c}\log M_{\mathrm{BH}, \mathrm{P} 06} \\
\text { (10) }\end{array}$ & $\begin{array}{c}\log M_{\mathrm{BH}, \mathrm{H} \alpha} \\
\text { (11) }\end{array}$ & $\begin{array}{c}\log M_{\mathrm{BH}, \mathrm{H} \beta} \\
\text { (12) }\end{array}$ \\
\hline PMNJ0134-0931 & 2.22 & 1410 & $5.1 \pm 0.5$ & $4.2 \pm 1.4$ & .. & & & & & & \\
\hline Q0142-100 & 2.7 & & $3.8 \pm 0.3$ & $2.7 \pm 0.6$ & 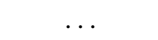 & $6.0 \pm 0.2$ & $6.5 \pm 1.3$ & 46.42 & 9.4 & $.4 \pm 0.3$ & $9.1 \pm 0.4$ \\
\hline SDSS0246-0825 & 1.69 & 540 & $2.5 \pm 0.2$ & $2.5 \pm 0.6$ & $2.9 \pm 0.1$ & $4.5 \pm 2.8$ & $5.0 \pm 0.2$ & $\ldots$ & $\ldots$ & & $\ldots$ \\
\hline MG0414+0534 & 2.6 & $\ldots$ & $5.3 \pm 0.3$ & $10.8 \pm 2.3$ & & & & 45.73 & 9.3 & $9.4 \pm 0.3$ & \\
\hline HS0810+2554 & 1.51 & 490 & $3.8 \pm 0.0$ & $4.4 \pm 1.1$ & $3.6 \pm 0.1$ & $4.6 \pm 0.4$ & $3.9 \pm 0.1$ & $\ldots$ & $\ldots$ & $\ldots$ & $\ldots$ \\
\hline SBS0909+523 & 1.38 & 410 & $3.1 \pm 0.6$ & $4.1 \pm 1.6$ & $3.5 \pm 0.5$ & $4.1 \pm 1.1$ & $\ldots$ & 45.96 & 9.6 & $9.0 \pm 0.3$ & $9.2 \pm 0.5$ \\
\hline FBQ0951+2635 & 1.25 & 290 & $6.3 \pm 0.4$ & $7.6 \pm 0.8$ & $6.0 \pm 0.3$ & $7.2 \pm 0.8$ & . & 45.48 & 8.9 & $9.4 \pm 0.3$ & $9.5 \pm 0.3$ \\
\hline Q0957+561 & 1.41 & 570 & $3.0 \pm 0.2$ & $3.3 \pm 0.9$ & $3.3 \pm 0.2$ & $5.2 \pm 0.5$ & .. & 46.25 & 9.3 & $9.1 \pm 0.3$ & $9.2 \pm 0.4$ \\
\hline $\mathrm{J} 1004+1229$ & 2.7 & $\ldots$ & $3.8 \pm 0.1$ & $5.9 \pm 1.5$ & $\ldots$ & $\ldots$ & & 46.12 & 9.3 & $9.3 \pm 0.3$ & $9.6 \pm 0.4$ \\
\hline HE1104-1805 & 2.32 & 970 & $4.7 \pm 0.2$ & $3.8 \pm 0.9$ & $\ldots$ & & $\ldots$ & 45.59 & 9.4 & $9.2 \pm 0.3$ & $8.9 \pm 0.4$ \\
\hline PG1115+080 & 1.7 & $\ldots$ & $4.0 \pm 0.1$ & $4.4 \pm 0.2$ & $4.0 \pm 0.2$ & $5.7 \pm 3.1$ & $6.7 \pm 1.0$ & 45.15 & 9.0 & $8.8 \pm 0.2$ & $8.8 \pm 0.3$ \\
\hline B $1152+200$ & 1.0 & $\ldots$ & $7.1 \pm 2.6$ & $\cdots$ & $\ldots$ & $\ldots$ & $3.3 \pm 0.6$ & . & & & $\ldots$ \\
\hline $\mathrm{H} 1413+117$ & 2.55 & 1650 & $5.3 \pm 0.8$ & $6.7 \pm 1.9$ & $\ldots$ & $8.0 \pm 3.2$ & $3.3 \pm 0.6$ & 45.67 & 8. & $9.3 \pm 0.3$ & $9.5 \pm 0.3$ \\
\hline B1422+231 & 3.63 & 2720 & & $6.1 \pm 2.2$ & $\ldots$ & & & 46.65 & 9.7 & & $9.9 \pm 0.4$ \\
\hline FBQ1633+3134 & 1.52 & 1720 & $4.1 \pm 0.7$ & $4.6 \pm 0.9$ & $4.1 \pm 0.5$ & $6.4 \pm 0.6$ & $3.8 \pm 0.7$ & 45.81 & 9.2 & $9.2 \pm 0.3$ & $9.2 \pm 0.6$ \\
\hline Q2237+030 & 1.70 & 1070 & $4.8 \pm 0.6$ & $3.8 \pm 1.4$ & $\ldots$ & $\ldots$ & $\ldots$ & $\ldots$ & $\ldots$ & $\ldots$ & $\ldots$ \\
\hline
\end{tabular}

Notes. Column 1: name; Column 2: redshift. Cases with three significant digits were measured from [O III], while cases with two come from the Castles website (http://www.cfa.harvard.edu/castles/). See P06 for references. Column 3: FWHM of [O III] $\lambda 5007 \AA$ line $\left(\mathrm{km} \mathrm{s}^{-1}\right)$, with the instrumental resolution of $\sigma_{*} \approx 36 \mathrm{~km} \mathrm{~s}^{-1}$ subtracted in quadrature. Column 4: $\mathrm{FWHM}_{\mathrm{H} \alpha}\left(10^{3} \mathrm{~km} \mathrm{~s}^{-1}\right)$ measured from the Triplespec data. Column 5: FWHM $\mathrm{H} \beta\left(10^{3} \mathrm{~km} \mathrm{~s}^{-1}\right)$ measured from the Triplespec data. Asterisks denote objects with incomplete spectral coverage of the $\mathrm{H} \beta$ line. Column $6: \mathrm{FWHM}_{\mathrm{MgII}}\left(10^{3} \mathrm{~km} \mathrm{~s}^{-1}\right) \mathrm{measured}$

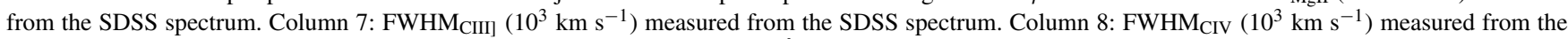
SDSS spectrum. Column 9: monochromatic luminosity ( $\mathrm{erg} \mathrm{s}^{-1}$ ) at $\lambda=5100 \AA ̊$ as measured from photometry (P06). Column 10: $\log \left(M_{\mathrm{BH}} / M_{\odot}\right)$ from P06; typically based on the $\mathrm{C}$ IV line. We do not quote errors in the BH masses, since systematic uncertainties dominate. We typically quote a factor of 4 error as derived by Vestergaard \& Peterson 2006. Column 11: $\log \left(M_{\mathrm{BH}} / M_{\odot}\right)$ calculated using Equation (1) and the FWHM $\mathrm{H}_{\alpha}$ from Column 4 combined with the luminosity from Column 9 . Note that the errors in the $\mathrm{H} \alpha$ masses shown here include only measurement uncertainties. Column 12 : $\log \left(M_{\mathrm{BH}} / M_{\odot}\right)$ based on $\mathrm{H} \beta$. In this case Equation (2) and $\mathrm{FWHM}_{\mathrm{H} \beta}$ from Column 5 are combined with the luminosity from Column 9.

2005a; Greene et al. 2009). The width of the narrow $\mathrm{H} \beta$ line (fit with a single Gaussian) is tied to that of [O III] whenever possible (Table 3). ${ }^{5}$ Finally, broad $\mathrm{H} \beta$ is fit with up to four (typ-

\footnotetext{
5 SBS0909+523 is the one exception. The narrow-line component is very strong in this object, even in the UV lines (see Figures 1 \& 2) and is generally narrower in other transitions than in the [O III] line. Interestingly, the broad lines appear to be redshifted compared to the narrow lines in this object. It would be worth attempting integral-field spectroscopy to investigate any spatial offsets corresponding to the observed velocity offset.
}

ically two) broad components. We also find the need to impose a lower limit of $1000 \mathrm{~km} \mathrm{~s}^{-1}$ on the broad components so that they do not erroneously fit a narrow-line component. We note that in these bright quasars the flux contribution from the narrow lines is typically small. Nevertheless, the treatment of the narrow lines is a source of uncertainty in the FWHM measurements, particularly in the UV. As described below, we therefore perform an additional fit with no narrow component and fold the difference into our total error budget. 

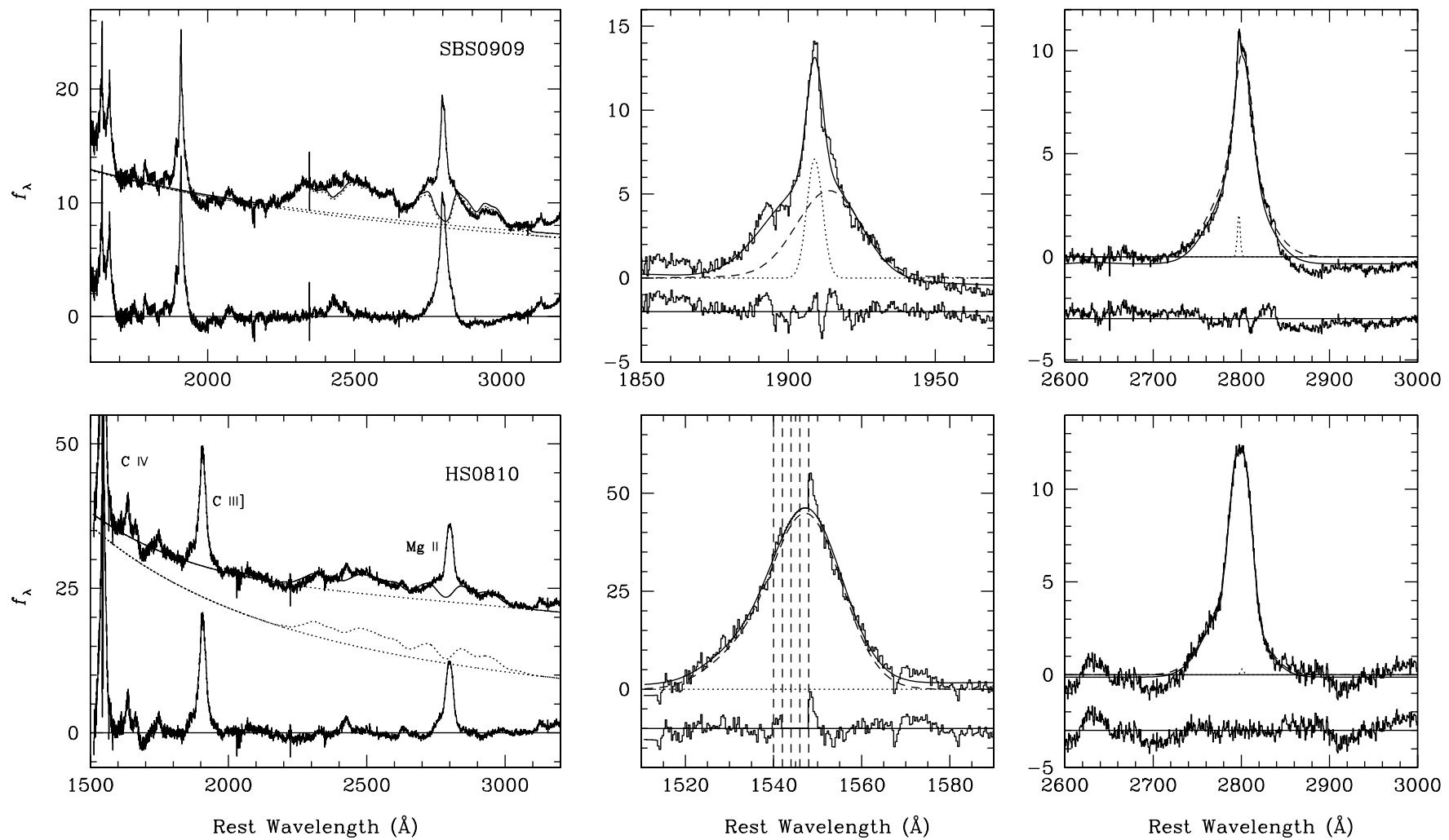

Figure 2. Example fits to the continuum (left), $\mathrm{C}_{\mathrm{IV}}$ or C $\mathrm{III}$ ] (middle), and Mg II (right) lines from the SDSS data, shown in units of $10^{-15} \mathrm{erg} \mathrm{s}^{-1} \mathrm{~cm}^{-2} \AA^{-1}$. We show the data (solid histogram), the total model (thin solid), the broad- (dashed) and narrow-line (dotted) model components, and residuals below (thin solid histogram). The dashed vertical lines denote a masked region of the spectrum that is heavily absorbed in the original spectrum. Note that in the C III] profile (top-middle panel) we show only the broad component of the C III] line itself.

Our approach is similar in the case of the $\mathrm{H} \alpha+[\mathrm{N}$ II] complex (see also Ho et al. 1997; Greene \& Ho 2004). Here we fix the narrow $\mathrm{H} \alpha$ and $[\mathrm{N}$ II] to the [O III] line width, fix the relative wavelengths to laboratory values, and fix the relative strengths of the [N II] $\lambda \lambda 6548,6584 \AA$ to $1: 3$. In cases where there is no [O III] line, we fix the narrow-line width to $500 \mathrm{~km} \mathrm{~s}^{-1}$. Generally in these cases the narrow components are too weak to be fit independently. Uncertainties in this procedure are estimated using an alternate fit with no narrow-line components. We fit the broad $\mathrm{H} \alpha$ with as many as four Gaussians, but do not tie them to the $\mathrm{H} \beta$ profile in any way. All of the Triplespec spectra and fits are shown in Figure 1 and the Appendix.

Our fits to the UV lines proceed in a similar fashion, with each broad line modeled as the sum of up to four Gaussians. We still choose to tie the narrow-line components to the width of [O III] as above, although we note that the proper treatment of the narrow component of $\mathrm{C}$ IV remains a matter of debate in the literature (Baskin \& Laor 2005; Vestergaard \& Peterson 2006; Kelly \& Bechtold 2007; Shen et al. 2008b). Again, in cases without available [O III] fits, we have simply chosen a representative width of $500 \mathrm{~km} \mathrm{~s}^{-1}$, and again we perform a second, narrow-line-free, fit. Finally, we include a linear continuum component to remove residual continuum errors (Figure 2). We note that the quality of the $\mathrm{Mg}$ II fit does depend on the Fe II subtraction, and our model for the Fe II continuum is poorly constrained directly beneath the Mg II line. However, numerous authors have now shown that the uncertainty in FWHM incurred by differing Fe II models is small $(<0.02$ dex; Salviander et al. 2007; Fine et al. 2008).

In addition to the standard Mg II and C IV lines, we have attempted to model the C III] 1909 transition, since it seems worth investigating every possible transition in the UV (and at least in one object, reverberation mapping with this line yielded a mass that is consistent with other transitions; Onken \& Peterson 2002). There are good reasons to avoid C III], including blending with S III] $\lambda 1892$, Al III $\lambda 1857$, and Fe III multiplets (e.g., Dietrich et al. 2002). To minimize these degeneracies, each of the three lines ( $\mathrm{CIII}_{\text {, }} \mathrm{S}$ III], and $\mathrm{Al}$ III) is modeled as a single Gaussian with the same width. The relative centroids are fixed to laboratory values and the strengths are left free. We include a narrow component only for $\left.\mathrm{C}_{\mathrm{III}}\right]$, and it is fixed to the [O III] width as above. Although we also attempted to include the Fe III multiplets (Vestergaard \& Wilkes 2001) in the fit, keeping the width constrained to that of the Fe II multiplets, the results were not well constrained. It can be seen in Table 3 that, unexpectedly, in three out of six cases FWHM $_{\mathrm{C}_{\mathrm{III}}}$ is larger than $\mathrm{FWHM}_{\mathrm{C} \text { Iv }}$. However, these are systems with narrow or broad absorption-line systems, which make the $\mathrm{C}$ IV fits particularly uncertain.

After fitting, our velocity measure of choice is a nonparametric full-width at half-maximum (FWHM) derived from our multi-Gaussian fits. We are aware that many authors advocate the use of the line dispersion rather than the FWHM (e.g., Peterson et al. 2004; Onken et al. 2004; Denney et al. 2009a). We do not believe there to be a strong argument in favor of one or the other line-width measurement at the current time (although see Collin et al. 2006), but we do know that our technique is robust in the presence of noisy spectra, even when fitting thousands of spectra. We direct the interested reader to the appendix of Greene \& Ho (2007a) for a detailed explication of our reasoning, but see also Denney et al. (2009a). 


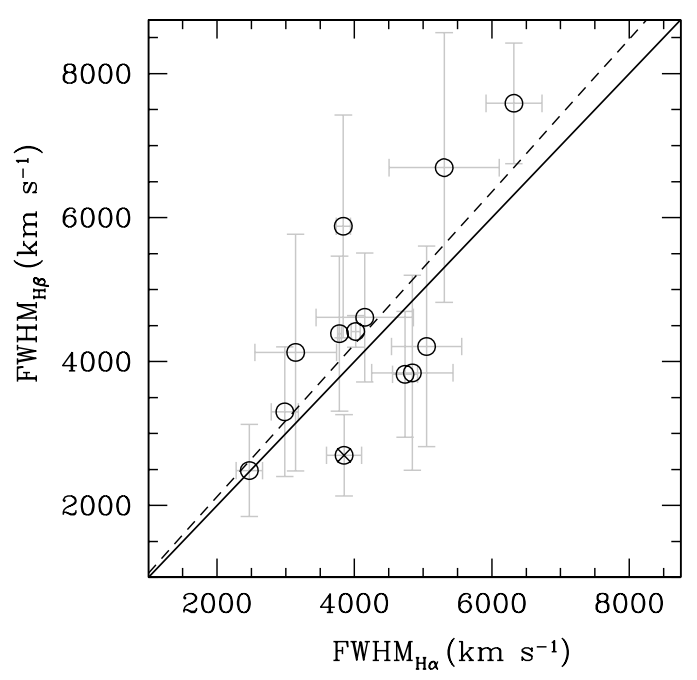

Figure 3. Comparison between the line widths derived from $\mathrm{H} \alpha$ and $\mathrm{H} \beta$ in the Triplespec data. $\mathrm{H} \beta$ lines with incomplete spectral coverage are noted with a cross. The one-to-one line (solid) is shown for reference only. As expected (e.g., Greene \& Ho 2005b) $\mathrm{H} \beta$ is generally broader than $\mathrm{H} \alpha$. Excluding the compromised $\mathrm{H} \beta$ fits, we find $\left\langle\mathrm{FWHM}_{\mathrm{H} \alpha} / \mathrm{FWHM}_{\mathrm{H} \beta}\right\rangle=0.9 \pm 0.2$ (dashed line), which is consistent with the $7 \%$ difference in line width found by Greene $\&$ Ho (2005b), albeit with considerable scatter. We note that MG0414+0534 has a very large and very unreliable $\mathrm{H} \beta$ line that is off the scale of this plot.

\subsection{Linewidth Comparisons}

In this subsection, we present figures comparing various estimates of line width derived from different elemental transitions (Figures 3-6). It is clear from these figures that measurement uncertainties alone lead to a significant amount of scatter and that the total dynamic range in line width for any transition is only a factor of $\lesssim 3$ (e.g., Fine et al. 2008; Shen et al. 2008b).

For each comparison figure, we have calculated the mean and standard deviation in the ratio of the two lines (not the error in the mean). These numbers are quoted in the figure captions. We have also calculated non-parametric correlation coefficients for each line pair. However, presumably because the samples are so small, none of the correlations are formally significant.

We cannot come to any strong conclusions based on these comparisons, due to both the small sample size and the large measurement errors. We confirm that $\mathrm{H} \beta$ is typically broader than $\mathrm{H} \alpha$ (Figure 3; e.g., Greene \& Ho 2005b, and references therein). It has long been known that high-ionization lines such as $\mathrm{C}$ IV are typically broadened and blueshifted compared to, e.g., Mg II and the Balmer lines (e.g., Gaskell 1982; Osterbrock \& Shuder 1982; Baldwin et al. 1996). While our observations are consistent with that trend (Figure 4), we cannot say much more than that. Over this limited range, we do not see strong evidence of a correlation between the widths of the Balmer lines and $\mathrm{C}_{\text {IV }}$, but to a large degree the scatter is driven by the difficulty in measuring a reliable width for the broad-absorption system in $\mathrm{H} 1413+117$. Furthermore, the (few) $\mathrm{C}$ III] measurements we have seem to be correlated with the Mg II line widths (Figure 4). We believe $\mathrm{C} \mathrm{III]} \mathrm{merits} \mathrm{investigation} \mathrm{with} \mathrm{a} \mathrm{much} \mathrm{larger} \mathrm{sample.}$ Finally, we tend to derive somewhat broader line widths than P06 from the UV lines, presumably because we have subtracted the Fe II emission, which P06 could not do (Figure 6). In a couple of cases the difference in narrow-line treatment also plays a role. We plan to revisit the UV-based mass estimates for the entire sample using modern spectroscopy (e.g., from SDSS) in future work.

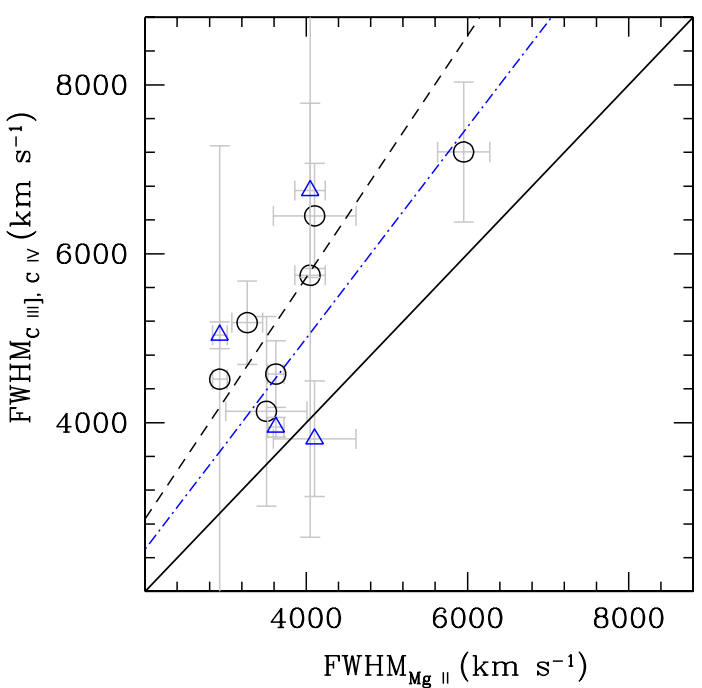

Figure 4. Comparison between the line widths derived from $\mathrm{Mg}_{\text {II }}$ and $\left.\mathrm{C}_{\mathrm{III}}\right]$ (black open circles) or CIV (blue open triangles) from the SDSS data. The one-to-one line (solid) is shown for reference only. We find $\left\langle\mathrm{FWHM}_{\mathrm{Mg}_{\text {II }}} /\right.$ $\left.\mathrm{FWHM}_{\mathrm{C} \text { III }}\right\rangle=0.7 \pm 0.1$ (black dashed line) and $\left\langle\mathrm{FWHM}_{\mathrm{Mg}_{\text {II }}} / \mathrm{FWHM}_{\mathrm{C}_{\text {IV }}}\right\rangle=$ $0.8 \pm 0.2$ (blue dot-dashed line).

(A color version of this figure is available in the online journal.)

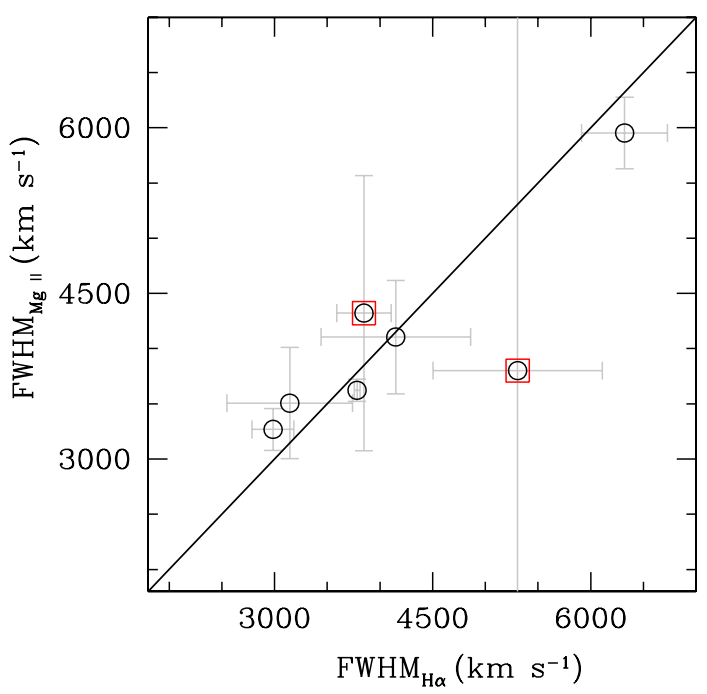

Figure 5. Comparison between the line widths derived from $\mathrm{H} \alpha$ and $\mathrm{Mg}$ III or a scaled C III] using a ratio of 0.7 (Figure 4) when necessary (red open squares). As above, we show the one-to-one relation as a solid line. Within the scatter, the two measures agree, with $\left\langle\mathrm{FWHM}_{\mathrm{H} \alpha} / \mathrm{FWHM}_{\mathrm{Mg}_{\mathrm{II}}}\right\rangle=1.0 \pm 0.2$.

(A color version of this figure is available in the online journal.)

\subsection{Mass Estimates}

The primary goal of our study is to remove any potential systematic bias in the C IV-based masses presented in P06 by calculating Balmer-based virial masses. In calculating $\mathrm{H} \alpha$-based $\mathrm{BH}$ masses, we start with the radius-luminosity relation of Bentz et al. (2009a). Since this relation was calibrated using $\mathrm{H} \beta$, we then convert $\mathrm{FWHM}_{\mathrm{H} \beta}$ to $\mathrm{FWHM}_{\mathrm{H} \alpha}$ using the relation derived in Greene \& Ho (2005b), which both increase the scaling with $\mathrm{FWHM}_{\mathrm{H} \alpha}$ to the 2.06 power and slightly changes the prefactor. Finally, for consistency with P06, we assume a scaling factor that is 1.8 times higher than the assumption of isotropic random 


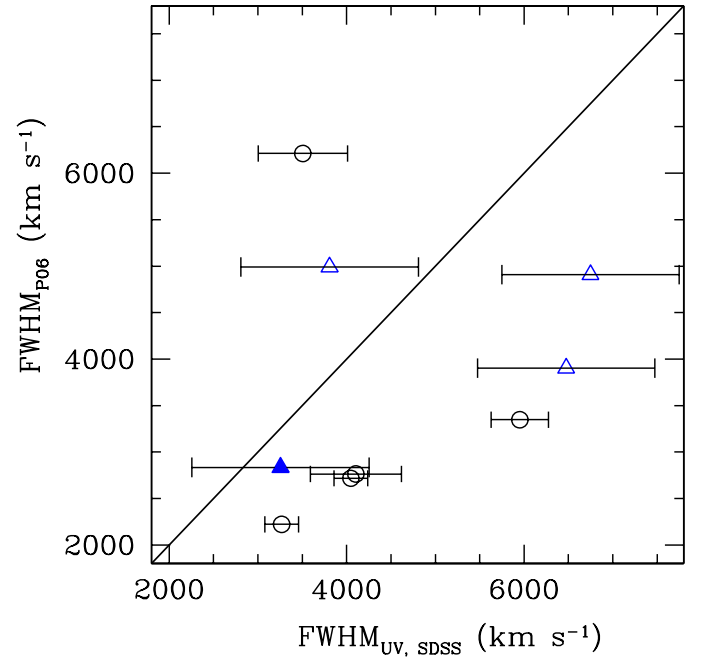

Figure 6. Comparison between our measured UV line widths from Mg II (black open circles) or C IV (blue open triangles) and those published in P06. Again, the scatter is quite large $\left\langle\mathrm{FWHM}_{\mathrm{Mg}_{\mathrm{II}}} / \mathrm{FWHM}_{\mathrm{P} 06}\right\rangle=1.5 \pm 0.5$, and again the unity relation is shown as a solid line. Our tendency to derive broader line widths than P06 derives from our continuum subtraction and treatment of the narrow lines. H1413+117 is highlighted as a filled square because the broad absorption feature in the $\mathrm{C}$ IV line makes fitting difficult.

(A color version of this figure is available in the online journal.)

motions, (e.g., Onken et al. 2004; Greene \& Ho 2006) yielding

$$
\begin{aligned}
M_{\mathrm{BH}}= & (9.7 \pm 0.5) \times 10^{6}\left(\frac{L_{5100 \AA}}{10^{44} \mathrm{erg} \mathrm{s}^{-1}}\right)^{0.519 \pm 0.07} \\
& \times\left(\frac{\mathrm{FWHM}_{\mathrm{H} \alpha}}{10^{3} \mathrm{~km} \mathrm{~s}^{-1}}\right)^{2.06 \pm 0.06}
\end{aligned}
$$

The masses calculated using this formalism are displayed in Table 3 and Figure 7(a). Note that in the case of B1422+231, $\mathrm{H} \alpha$ is outside our observing window. In this case, we use the
$\mathrm{H} \beta$ line and the following (very similar) formalism:

$$
\begin{aligned}
M_{\mathrm{BH}}= & (9.1 \pm 0.5) \times 10^{6}\left(\frac{L_{5100 \AA}}{10^{44} \mathrm{erg} \mathrm{s}^{-1}}\right)^{0.519 \pm 0.07} \\
& \times\left(\frac{\mathrm{FWHM}_{\mathrm{H} \beta}}{10^{3} \mathrm{~km} \mathrm{~s}^{-1}}\right)^{2} .
\end{aligned}
$$

The $\mathrm{H} \beta$ masses are presented in Figure 7(b).

The luminosities used here are derived from the Hubble Space Telescope (HST) photometry of the CASTLES sample, as described in detail in P06. Briefly, the quasar and host galaxy images are optimized along with the lens model using LENSFIT (a variant of GALFIT; Peng et al. 2002). The quasars are modeled as point sources, the galaxies as Sérsic (1968) functions, and the lensing masses as singular isothermal ellipsoids. The demagnified and deblended $V, I, H$ quasar magnitudes are modeled as a power law, with the slopes presented in Table 2, and the luminosities come from this fit. We note that our spectroscopically derived slopes agree nicely with those from broadband photometry. We find $\left\langle\alpha_{\mathrm{opt}} / \alpha_{\mathrm{P} 06}\right\rangle=$ $1 \pm 0.5$, where $f_{\lambda} \propto \lambda^{\alpha}$. The $H S T H$-band images correspond closely to rest-frame $V$-band at $z \approx 2$, so the luminosities are very insensitive to uncertainties in the power-law slope.

We find a mean ratio of $\left\langle\log \left(M_{\mathrm{H} \alpha} / M_{\mathrm{P} 06}\right)\right\rangle=-0.02 \pm 0.5$ between the two mass estimates. Although the scatter is large, we find no evidence for a systematic offset in the C IV-based masses. Therefore, to the extent that virial mass estimates have merit in this mass, luminosity, and redshift regime (as yet untested directly), our new spectroscopic observations confirm the results presented in $\mathrm{P} 06$.

\subsection{Uncertainties}

Uncertainties in line widths are difficult to estimate. In our case, the signal-to-noise $(\mathrm{S} / \mathrm{N})$ ratios of the spectra are not very high (see Table 1), which contributes substantially to the error budget. We use Monte Carlo simulations to estimate the magnitude of the uncertainties due to finite $\mathrm{S} / \mathrm{N}$. For each observed line, we generate 1000 mock spectra with the same
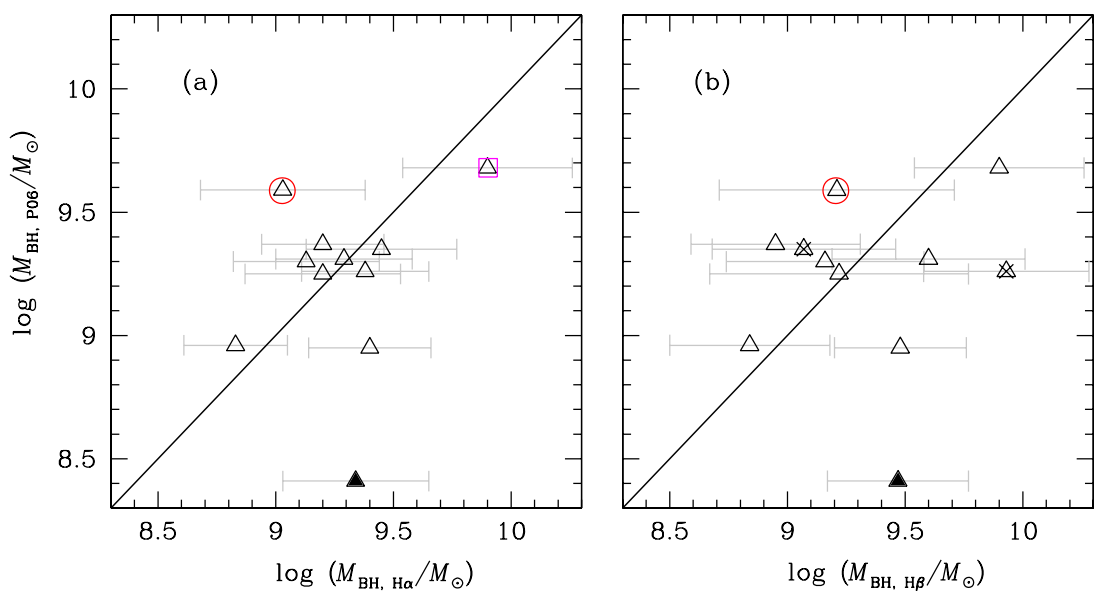

Figure 7. (a) We compare $\mathrm{BH}$ mass estimates based on the $\mathrm{H} \alpha$ transition with those from P06. The P06 masses are based on C IV with one exception (red open circle; $\mathrm{Mg}$ II) while our masses are based on $\mathrm{H} \alpha$ with one exception (magenta open square; $\mathrm{H} \beta$ ). The unity relation (solid line) is shown to guide the eye. While the two mass estimates are not strongly correlated, we do not find any evidence for a systematic offset between the two (the median $M_{\mathrm{H} \alpha} / M_{\mathrm{P} 06}=1.0 \pm 0.4$ ). $\mathrm{H} 1413+117$, due to its broad-absorption system, is highlighted as a filled symbol. (b) Same as (a), except using $\mathrm{H} \beta$ as the virial indicator. In this case, objects with only partial observations of the $\mathrm{H} \beta$ line are highlighted with crosses. As above the $\mathrm{P} 06$ mass that is based on $\mathrm{Mg}_{\mathrm{II}}$ is identified with a red circle. We find a median $M_{\mathrm{H} \beta} / M_{\mathrm{P} 06}=1.0 \pm 0.5$. The filled triangle is $\mathrm{H} 1413+117$.

(A color version of this figure is available in the online journal.) 

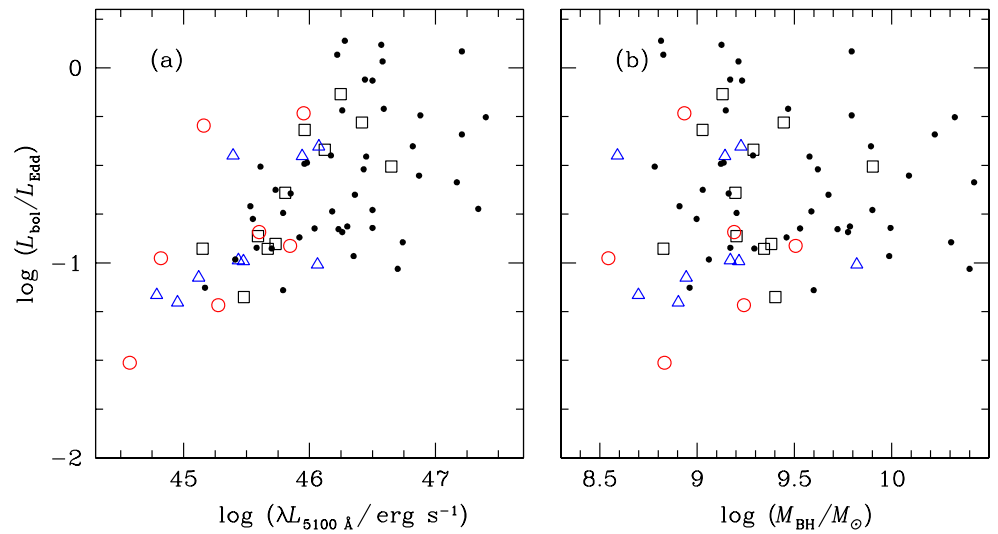

Figure 8. (a) We reproduce Figure 2 from Netzer et al. (2007, black dots; based on $H \beta$ ) and include the targets from this study (large black open squares), as well as the entire P06 sample, differentiating between Mg II-based (small red circles) and C IV-based (small blue triangles) masses. Here we plot monochromatic $5100 \AA$ luminosity vs. the Eddington ratio. We follow Netzer et al. and assume that the bolometric luminosity is 7 times $L_{5100 \AA}$. Both our mass estimates and those from Netzer et al. are based on Balmer emission lines ( $\mathrm{H} \beta$ in their case). (b) $M_{\mathrm{BH}}$ vs. $L_{\text {bol }} / L_{\mathrm{Edd}}$; symbols as in (a). We see that the distributions in each diagram are similar between the two samples, although the lensed systems extend to objects that are factors of $2-3$ times fainter.

(A color version of this figure is available in the online journal.)

shape as our best-fit model and the same noise as our observed spectrum. In order to model residual uncertainties in continuum subtraction, we include a broadened Fe II spectrum with an amplitude that scatters around $10 \%$ of the peak flux. Using the same procedures as in the real data, we then measure the bestfit parameters for each of these simulations. The uncertainty is determined from the distribution of simulated line dispersion to be half of the width encompassing $68 \%$ of the simulated galaxy measurements, and ranges from $\sim 10 \%$ to $40 \%$ of the measured value. Now, in addition to noise, the treatment of narrow emission lines can be a significant cause of systematic uncertainty. Thus, we perform a second fit with the narrow component of each line turned off, and the difference between the two represents a second estimate of the uncertainty. We adopt the larger of these as our final error in line width.

At the same time, quasar variability adds additional uncertainty, since the rest-frame optical and UV spectra analyzed here were not obtained contemporaneously. Wilhite et al. (2007) and Denney et al. (2009a) both find that variability in line width tends to be small $(\sim 30 \%)$ and thus only contributes $\sim 0.1$ dex scatter to BH mass estimates. Another feature of quasar spectra that complicates emission-line-width measurements is the presence of blueshifted absorption features with velocity widths of tens (narrow) to tens of thousands (broad) of $\mathrm{km} \mathrm{s}^{-1}$. Both H1413+117 and (to a lesser extent) PG1115+080 display broad absorption features, while Q0957+561 and HS0810+2554 have significant narrow absorption. In these cases, we simply mask the regions from the fit, but note that particularly in the case of H1413+117, our ability to measure a reliable C IV width is seriously compromised, since even the line center is not well defined.

In addition to the line widths presented here, the $\mathrm{BH}$ masses depend on the luminosity of the quasar. We briefly review the arguments that the lens modeling does not add significant uncertainties to our results and direct the interested reader to Appendix B of P06 for more details. The primary source of uncertainty in the models is that gravitational time delay and lensing substructures can lead to anomalous quasar magnification ratios, which translate into an uncertainty in the quasar luminosity. However, this error should be no larger than 0.1$0.2 \mathrm{mag}$, and thus does not contribute significantly to the $\mathrm{BH}$ mass uncertainty. Actual measurement uncertainties from model fitting are tiny for the quasar.
In Table 3, we present the formal uncertainties in $\mathrm{BH}$ mass arising from errors in the FWHM measurements and the formal uncertainty in the slope of the radius-luminosity relation. We wish to emphasize that the true errors in $\mathrm{BH}$ mass are probably dominated by systematic uncertainties arising from our ignorance of the structure and kinematics of the BLR that translate into errors in inferring both its true extent and velocity field based on the observations (Krolik 2001; Collin et al. 2006; Greene \& Ho 2006). At present, we really have no concrete confirmation that (1) the same radius-luminosity relation applies to quasars at these luminosities (although see Kaspi et al. 2007) nor that (2) it is meaningful to assume that the BLR gas is in virial equilibrium and not, for instance, dominated by a massive outflow (e.g., Baldwin et al. 1996; Richards et al. 2002a; Leighly \& Moore 2004; Fine et al. 2008).

\section{DEMOGRAPHICS OF LENSED QUASARS}

To summarize, we have obtained $\mathrm{H} \alpha$-based $\mathrm{BH}$ masses for a large fraction of the lensed quasars with measured host galaxy luminosities from $\mathrm{P} 06$. While the scatter between $\mathrm{H} \alpha$-based and C IV- or $\mathrm{Mg}$ II-based $\mathrm{BH}$ masses is quite large $(\sim 0.5 \mathrm{dex})$, we do not see evidence for a systematic bias in mass. Thus, we do not alter the result of P06 that $M_{\mathrm{BH}}-L_{\text {bulge }}$ relations appear to evolve with cosmic time.

It is instructive to now compare the properties of the lensed sample with the general quasar population at $1 \lesssim z \lesssim 4$. A large number of studies have looked at the distributions of $\mathrm{BH}$ mass and Eddington ratio of luminous quasars (e.g., McLure \& Dunlop 2004; Kollmeier et al. 2006; Netzer et al. 2007; Fine et al. 2008; Shen et al. 2008b; Gavignaud et al. 2008) including some work on narrow-line quasars (Greene et al. 2009; Liu et al. 2009) as well as mass functions for quasars (e.g., Greene \& Ho 2007b; Vestergaard et al. 2008; Kelly et al. 2009). In most cases, the BH masses are based on C IV and $\mathrm{Mg}$ II from observed optical spectra. Generally, all works find the same surprisingly narrow range in measured line width, and correspondingly narrow distributions in $M_{\mathrm{BH}}$ and $L_{\mathrm{bol}} / L_{\mathrm{Edd}}$ at all redshifts (although as Gavignaud et al. point out, the derived distribution is quite sensitive to the assumed slope in the radiusluminosity relation).

For our purpose, it is easiest to compare with the results of Netzer et al., as their study is also based on $\mathrm{H} \beta$ observations. 

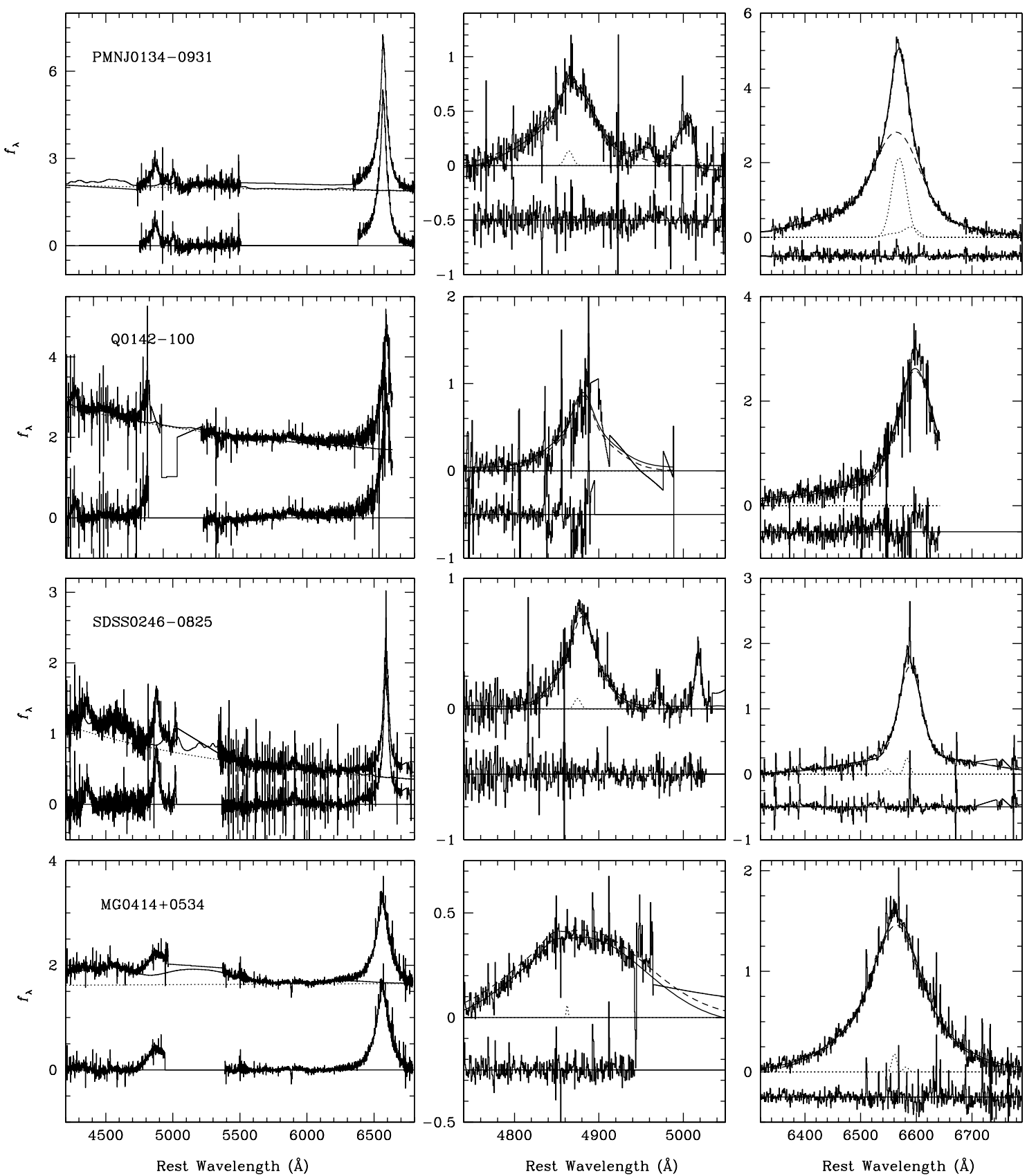

Figure A1. Fits to the continuum (left), $\mathrm{H} \beta$ (middle), and $\mathrm{H} \alpha$ (right) lines from the Triplespec data that do not appear in Figure 1. We show the data (solid histogram), the total model (thin solid), the broad- (dashed) and narrow-line (dotted) model components, and residuals below (thin solid histogram). Data are plotted with an arbitrary scale in $f_{\lambda}$. Spectral regions that are masked in the fit do not appear in the residuals (e.g., the red wing of $\mathrm{H} \beta$ for Q0142-100). Also note that in a couple of cases the [O III] fit is based exclusively on the $\lambda 4959$ line; no redshifts are derived for these targets.

For convenience, we adopt their assumption that $L_{5100 \AA}$ is $\sim$ one seventh of the bolometric luminosity and we take the Eddington luminosity to be $1.26 \times 10^{38}\left(M_{\mathrm{BH}} / M_{\odot}\right) \mathrm{erg} \mathrm{s}^{-1}$. Despite completely different selection criteria, the distributions in luminosity, and correspondingly inferred $\mathrm{BH}$ mass and Eddington ratio, are quite similar across the two samples (Figure 8). For the Triplespec sample, the median $\left\langle M_{\mathrm{BH}}\right\rangle \approx$ $2 \times 10^{9} M_{\odot}$ and the median $L_{5100 \AA} \approx 6 \times 10^{45} \mathrm{erg} \mathrm{s}^{-1}$ yield a typical Eddington fraction of $\sim 30 \%$. If we take the entire P06 sample, using $\mathrm{H} \alpha$-based masses when available, we find a median mass of $\sim 10^{9} M_{\odot}$, a median $L_{5100 \AA}$ of $2 \times 10^{45} \mathrm{erg} \mathrm{s}^{-1}$, and a median Eddington ratio of $10 \%$.

Unlike other studies, Netzer et al. actually argue that their sample displays a broad distribution in the Eddington ratio when compared to theoretical expectations that luminous quasars should be most readily observed at or near their Eddington luminosities (e.g., Marconi et al. 2004; Merloni 2004; Hopkins et al. 2006). We find the observed distributions unexpected for a somewhat different reason, namely the uniformly high $\mathrm{BH}$ masses. Assuming that the Eddington limit strictly applies and given the lower flux limit of the lensed sample, we could detect BHs with masses as low as $\sim 10^{8} M_{\odot}$. Furthermore, our intuition from the local BH mass function (e.g., Yu \& Tremaine 2002; Marconi et al. 2004) suggests that $10^{8} M_{\odot}$ BHs ought to be far more numerous than $10^{9} M_{\odot}$ systems due to the exponential decline in the space density of the most luminous galaxies. Given that supercritical accretion may be observed locally (e.g., Pounds et al. 1995; Mineshige et al. 2000; Desroches et al. 

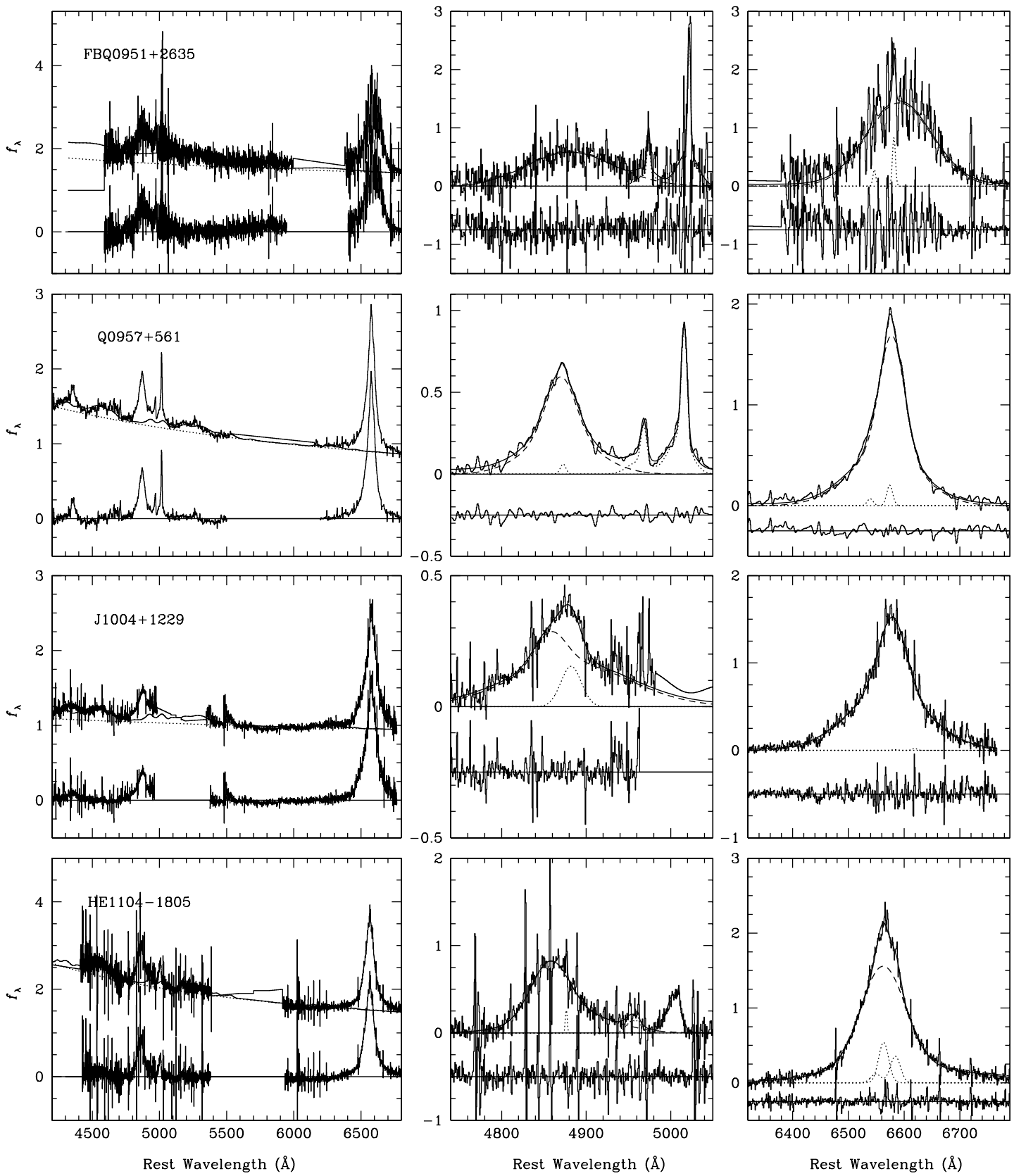

Figure A1. (Continued)

2009), we find the preponderance of $\sim 10^{9} M_{\odot}$ BHs radiating at $\sim 10 \%$ of their Eddington luminosity to be noteworthy.

We can be slightly more quantitative. Following, e.g., Somerville (2009), we can transform the observed $z \sim 2$ galaxy mass function (e.g., Fontana et al. 2006) into the expected $\mathrm{BH}$ mass function. For reference, at the median luminosity of the Triplespec sample, the Eddington limit sets a bound of $\sim 4 \times 10^{8} M_{\odot}$ on observable BHs. If the relation between $M_{\mathrm{BH}}$ and galaxy mass were identical to what it is today (e.g., Gültekin et al. 2009), then the BHs with mass $\approx 4 \times 10^{8} M_{\odot}$ ought to be $\sim 50$ times more common than the median observed mass of $M_{\mathrm{BH}} \approx 2 \times 10^{9} M_{\odot}$. Note that we are conservatively adopting the median rather than the minimum observed luminosity and assuming that the Eddington luminosity applies strictly. Taking the observed masses at the face value for the moment, and assuming that the selection of lensed quasars is simply a random selection of optically luminous quasars at $1<z<4$, we infer that either BHs radiating at 30\% of Eddington are 50 times more numerous than Eddington-limited objects or that the zero point in the $M_{\mathrm{BH}}-L_{\text {bulge }}$ relation has evolved by a factor of $\sim 3$ to the present day. This latter, of course, is the suggestion made by $\mathrm{P} 06$.

Obviously, such constraints are not particularly stringent at the moment. For one thing, the selection of lensed quasars is complicated to model, involving radio selection in some cases (which will tend to bias samples toward more massive systems; e.g., Heckman 1983; Mandelbaum et al. 2009). Furthermore, as pointed out by Somerville, joint constraints from galaxy and quasar luminosity functions on their own cannot distinguish between zero-point evolution or increased scatter in BH-bulge relations at high redshift. Finally, we do not know that the quasar duty cycle is independent of mass. Nevertheless, we cannot help but wonder whether the narrow range in observed line width, and the unexpectedly high average $\mathrm{BH}$ mass, do not instead 

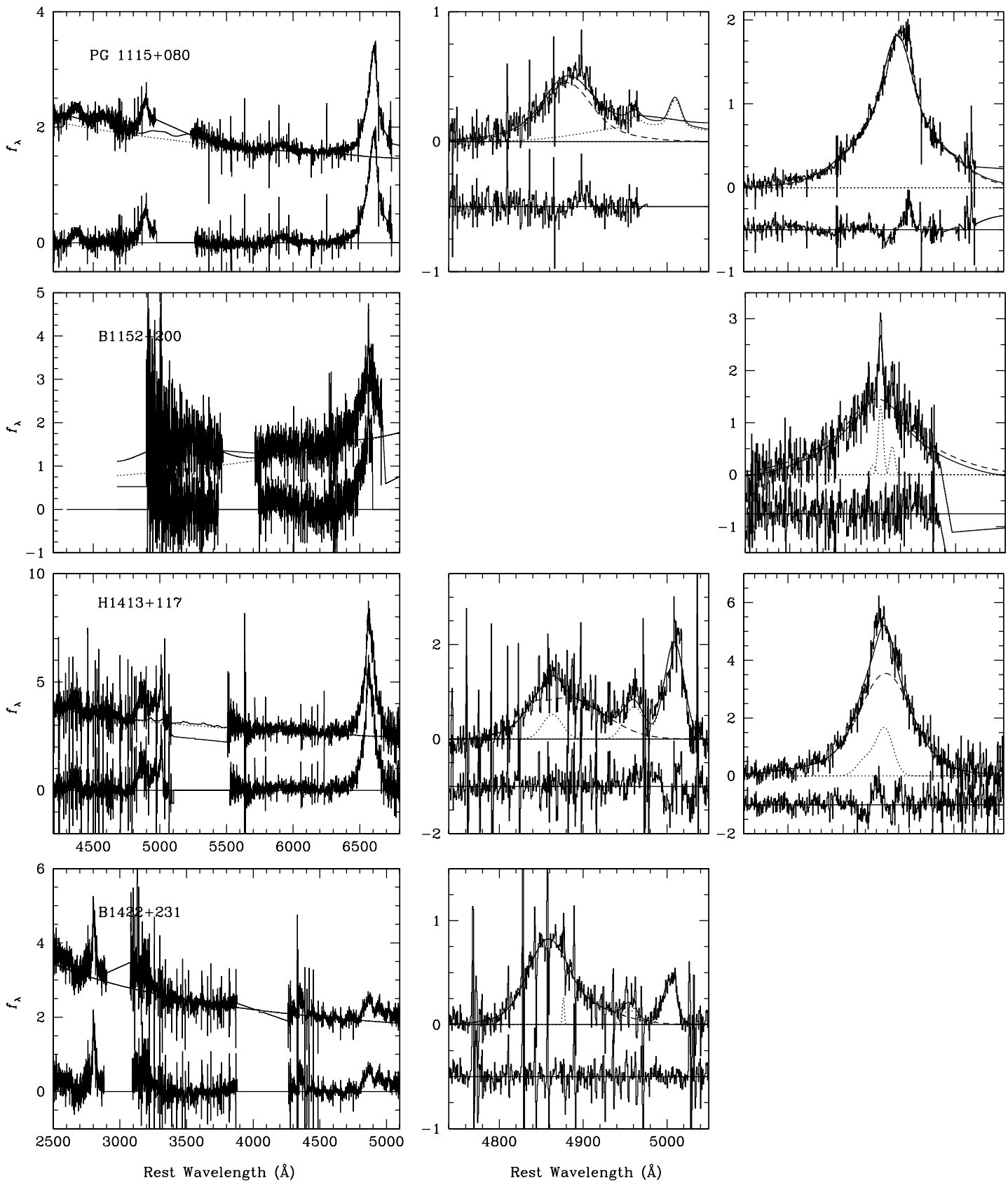

Figure A1. (Continued)

indicate a problem in virial mass estimators at high luminosity that may be resolved with better understanding of the physics of broad-line quasars.

Ultimately, the question is whether or not the broad emission lines in luminous quasars are dominated by virial motions. Of course, there is evidence for a non-virialized component in the C IV line (e.g., Baldwin et al. 1996; Richards et al. 2002a). On the other hand, there is clear evidence for virialized motion in the BLRs of at least a few nearby lower luminosity active galaxies (e.g., Peterson \& Wandel 1999; Onken \& Peterson 2002; Peterson et al. 2004). Also, the local virial masses seem to correlate with both $\sigma_{*}$ (e.g., Shen et al. 2008a) and $L_{\text {bulge }}$ (e.g., Kim et al. 2008b; Bentz et al. 2009b). Unfortunately, similar analysis is not yet available in the luminosity range of interest to us. At present, all we can say is that the CIVbased masses alone are not causing a net bias in the virial masses relative to the Balmer lines. True evolution in $\mathrm{BH}$-bulge scaling relations is by no means certain; far more pernicious sources of uncertainty remain, including potential biases in sample selection. At the minimum, to mitigate these concerns, quasar samples with identical selection at multiple redshifts are needed.

In closing, we note that $\mathrm{BH}-$ bulge relations are not the only ones purported to display unexpected evolution since a redshift $z \approx 2$. Recent work has shown that, at a fixed mass, elliptical galaxies were a factor of 2-5 smaller at redshifts of one and two, respectively, than they are today (e.g., Trujillo et al. 2006; van Dokkum et al. 2008; Franx et al. 2008; van der Wel et al. 2008; Damjanov et al. 2009). At first it seems counterintuitive that the most massive elliptical galaxies, which seem to have formed the bulk of their stars rapidly at an earlier epoch (e.g., Thomas et al. 2005), should grow less dense with time. However, a large number of minor mergers can very efficiently build the outskirts of elliptical galaxies (e.g., Boylan-Kolchin \& Ma 2007; 

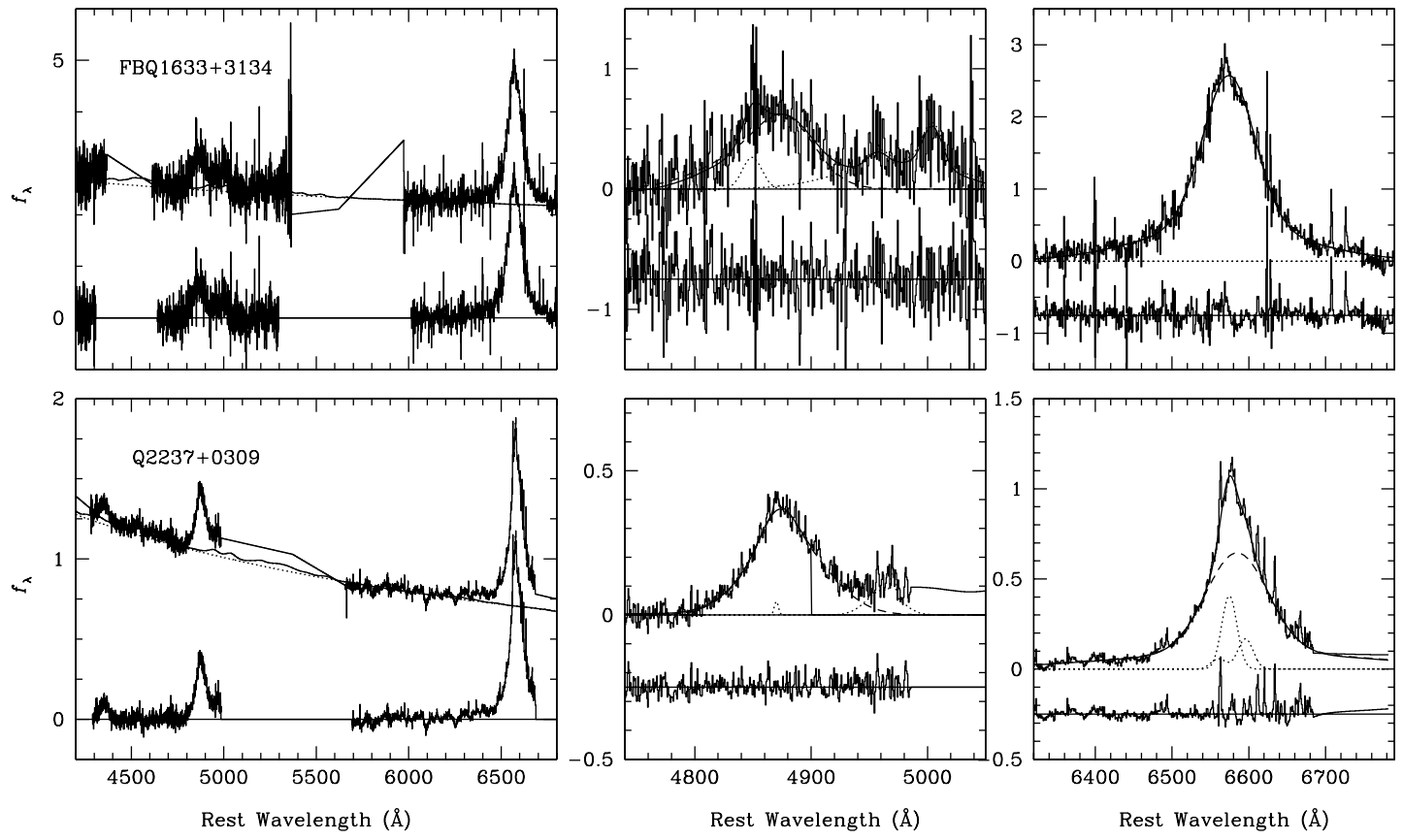

Figure A1. (Continued)

Bezanson et al. 2009; Naab et al. 2009). Interestingly, these gas-free minor mergers would need to grow the total galaxy mass by factors of 2-3 in order to match local observations (e.g., Bezanson et al. 2009). This is possible under a scenario where the BH-to-TOTAL stellar mass relation is steeper than linearity in low-mass galaxies, which is observed in nearby galaxies (Gültekin et al. 2009; Greene et al. 2008). In gas-free merging, there is no corresponding $\mathrm{BH}$ growth via accretion, which could facilitate a boost in the $\mathrm{BH}$-bulge ratio at late times (see also Hopkins et al. 2009). As argued by Peng (2007), numerous minor mergers will tend to drive the ratio of $\mathrm{BH}$ to galaxy mass toward a value of unity slope (e.g., his Figure 2(b) and 4(a)). Alternatively, as suggested by both Croton (2006) and Jahnke et al. (2009), it may be that disk components are common in the high-redshift galaxies but are subsequently subsumed into the main elliptical galaxy body. We mention this apparent coincidence in passing because it is intriguing, although we note that a variety of outstanding uncertainties are yet to be explored as far as the structural evolution of elliptical galaxies is concerned.

\section{SUMMARY}

We revisit the $\mathrm{BH}$ mass estimates for a sample of lensed quasars with high-fidelity host-galaxy luminosities from Peng et al. (2006b). While the Balmer-based masses presented here are arguably more robust than the UV-based estimates, we find no evidence for a systematic difference in $\mathrm{BH}$ masses based on the two methods. If we can take the Balmer-based virial masses at the face value, then we confirm the result of Peng et al. (2006b) that BHs appear to be overly massive relative to their hosts at high redshift. Intriguingly, the minor mergers that are invoked to puff up compact elliptical galaxies at late times would be very effective at boosting the galaxy to $\mathrm{BH}$ mass ratio as well. However, as pointed out many times, the persistently narrow range in observed line width for luminous quasars at high redshift provides substantial cause for concern in the veracity of the virial masses. Furthermore, it is not clear that we have yet assembled consistent comparison quasar samples across cosmic time with which to compare the $\mathrm{BH}$-bulge scaling relations. Our work is a necessary, but not sufficient, step in determining the true evolution of $\mathrm{BH}$-bulge relations with cosmic time.

The referee provided a very careful and insightful reading that substantively improved the manuscript. Both J.E.G. and C.Y.P. are grateful for many stimulating conversations with L.C.Ho, and we thank A. J. Barth and C. A. Onken for very useful comments. We thank M. Skrutskie for taking commissioning data for this project and for invaluable help with the reduction software. We thank H. Netzer for kindly providing his data table for Figure 8. Support for J.E.G. was partially provided by NASA through Hubble Fellowship grant HF-01196 awarded by the Space Telescope Science Institute, which is operated by the Association of Universities for Research in Astronomy, Inc., for NASA, under contract NAS 5-26555.

\section{APPENDIX}

\section{TRIPLESPEC SPECTRA}

In Figure A1 $(\mathrm{a}-\mathrm{d})$, we present the rest of the Triplespec spectra for completeness. Note that the Triplespec spectra for SBS0909 and HS0810 are shown in Figure 1.

\section{REFERENCES}

Abazajian, K. N., et al. 2009, ApJS, 182, 543

Alexander, D. M., et al. 2008, AJ, 135, 1968

Baldwin, J. A., et al. 1996, ApJ, 461, 664

Baskin, A., \& Laor, A. 2005, MNRAS, 356, 1029

Bentz, M. C., Peterson, B. M., Netzer, H., Pogge, R. W., \& Vestergaard, M. 2009a, ApJ, 697, 160

Bentz, M. C., Peterson, B. M., Pogge, R. W., \& Vestergaard, M. 2009b, ApJ, 694, L166

Bentz, M. C., Peterson, B. M., Pogge, R. W., Vestergaard, M., \& Onken, C. A. 2006, ApJ, 644, 133

Bentz, M. C., et al. 2009c, ApJ, 705, 199

Bezanson, R., van Dokkum, P. G., Tal, T., Marchesini, D., Kriek, M., Franx, M., \& Coppi, P. 2009, ApJ, 697, 1290

Blandford, R. D., \& McKee, C. F. 1982, ApJ, 255, 419

Boroson, T. A. 2003, ApJ, 585, 647 
Boroson, T. A., \& Green, R. F. 1992, ApJS, 80, 109

Boylan-Kolchin, M., \& Ma, C.-P. 2007, MNRAS, 374, 1227

Collin, S., Kawaguchi, T., Peterson, B. M., \& Vestergaard, M. 2006, A\&A, 456, 75

Croton, D. J. 2006, MNRAS, 369, 1808

Cushing, M. C., Vacca, W. D., \& Rayner, J. T. 2004, PASP, 116, 362

Damjanov, I., et al. 2009, ApJ, 695, 101

Denney, K. D., Peterson, B. M., Dietrich, M., Vestergaard, M., \& Bentz, M. C. 2009a, ApJ, 692, 246

Denney, K. D., et al. 2009b, ApJ, 704, L80

Desroches, L.-B., Greene, J. E., \& Ho, L. C. 2009, ApJ, 698, 1515

Dibai, E. A. 1980, Sov. Astron., 24, 389

Dietrich, M., Mathur, S., Grupe, D., \& Komossa, S. 2009, ApJ, 696, 1998

Dietrich, M., et al. 2002, ApJ, 581, 912

Ferrarese, L., Pogge, R. W., Peterson, B. M., Merritt, D., Wandel, A., \& Joseph, C. L. 2001, ApJ, 555, L79

Fine, S., et al. 2008, MNRAS, 390, 1413

Fontana, A., et al. 2006, A\&A, 459, 745

Fowler, A. M., \& Gatley, I. 1990, ApJ, 353, L33

Franx, M., van Dokkum, P. G., Schreiber, N. M. F., Wuyts, S., Labbé, I., \& Toft, S. 2008, ApJ, 688, 770

Gaskell, C. M. 1982, ApJ, 263, 79

Gaskell, C. M. 2009, ApJ, submitted (arXiv:0908.0328)

Gavignaud, I., et al. 2008, A\&A, 492, 637

Gebhardt, K., et al. 2000, ApJ, 543, L5

Grandi, S. A. 1982, ApJ, 255, 25

Greene, J. E., \& Ho, L. C. 2004, ApJ, 610, 722

Greene, J. E., \& Ho, L. C. 2005a, ApJ, 627, 721

Greene, J. E., \& Ho, L. C. 2005b, ApJ, 630, 122

Greene, J. E., \& Ho, L. C. 2006, ApJ, 641, L21

Greene, J. E., \& Ho, L. C. 2007a, ApJ, 670, 92

Greene, J. E., \& Ho, L. C. 2007b, ApJ, 667, 131

Greene, J. E., Ho, L. C., \& Barth, A. J. 2008, ApJ, 688, 159

Greene, J. E., Zakamska, N. L., Liu, X., Barth, A. J., \& Ho, L. C. 2009, ApJ, 702,441

Gültekin, K., et al. 2009, ApJ, 698, 198

Heckman, T. M. 1983, ApJ, 273, 505

Heckman, T. M., Miley, G. K., van Breugel, W. J. M., \& Butcher, H. R. 1981, ApJ, 247, 403

Ho, L. C. 2007, ApJ, 669, 821

Ho, L. C. 2009, ApJ, 699, 638

Ho, L. C., Darling, J., \& Greene, J. E. 2008, ApJ, 681, 128

Ho, L. C., Filippenko, A. V., Sargent, W. L. W., \& Peng, C. Y. 1997, ApJS, 112 , 391

Hopkins, P. F., Bundy, K., Hernquist, L., Wuyts, S., \& Cox, T. J. 2009, MNRAS, in press (arXiv:0909.2039)

Hopkins, P. F., Hernquist, L., Cox, T. J., Di Matteo, T., Robertson, B., \& Springel, V. 2006, ApJS, 163, 1

Horne, K. 1986, PASP, 98, 609

Jahnke, K., et al. 2009, ApJ, 706, L215

Kaspi, S., Brandt, W. N., Maoz, D., Netzer, H., Schneider, D. P., \& Shemmer, O. 2007, ApJ, 659, 997

Kaspi, S., Maoz, D., Netzer, H., Peterson, B. M., Vestergaard, M., \& Jannuzi, B. T. 2005, ApJ, 629, 61

Kelly, B. C., \& Bechtold, J. 2007, ApJS, 168, 1

Kelly, B. C., Vestergaard, M., \& Fan, X. 2009, ApJ, 692, 1388

Kim, M., Ho, L. C., Peng, C. Y., Barth, A. J., \& Im, M. 2008a, ApJS, 179, 283

Kim, M., Ho, L. C., Peng, C. Y., Barth, A. J., Im, M., Martini, P., \& Nelson, C. H. 2008b, ApJ, 687, 767

Kollmeier, J. A., et al. 2006, ApJ, 648, 128

Kormendy, J., \& Richstone, D. 1995, ARA\&A, 33, 581

Krolik, J. H. 2001, ApJ, 551, 72

Lauer, T. R., Tremaine, S., Richstone, D., \& Faber, S. M. 2007, ApJ, 670, 249

Leighly, K. M., \& Moore, J. R. 2004, ApJ, 611, 107

Liu, X., Zakamska, N. L., Greene, J. E., Strauss, M. A., Krolik, J. H., \& Heckman, T. M. 2009, ApJ, 702, 1098

Mandelbaum, R., Li, C., Kauffmann, G., \& White, S. D. M. 2009, MNRAS, 393, 377

Marconi, A., Risaliti, G., Gilli, R., Hunt, L. K., Maiolino, R., \& Salvati, M. 2004, MNRAS, 351, 169

McLeod, K. K., \& Bechtold, J. 2009, ApJ, 704, 415

McLure, R. J., \& Dunlop, J. S. 2004, MNRAS, 352, 1390

Merloni, A. 2004, MNRAS, 353, 1035

Mineshige, S., Kawaguchi, T., Takeuchi, M., \& Hayashida, K. 2000, PASJ, 52 , 499
Miralda-Escudé, J., \& Kollmeier, J. A. 2005, ApJ, 619, 30

Murray, N., Quataert, E., \& Thompson, T. A. 2005, ApJ, 618, 569

Naab, T., Johansson, P. H., \& Ostriker, J. P. 2009, ApJ, 699, L178

Nelson, C. H., Green, R. F., Bower, G., Gebhardt, K., \& Weistrop, D. 2004, ApJ, 615,652

Nelson, C. H., \& Whittle, M. 1996, ApJ, 465, 96

Netzer, H., Lira, P., Trakhtenbrot, B., Shemmer, O., \& Cury, I. 2007, ApJ, 671, 1256

Onken, C. A., Ferrarese, L., Merritt, D., Peterson, B. M., Pogge, R. W., Vestergaard, M., \& Wandel, A. 2004, ApJ, 615, 645

Onken, C. A., \& Kollmeier, J. A. 2008, ApJ, 689, L13

Onken, C. A., \& Peterson, B. M. 2002, ApJ, 572, 746

Osterbrock, D. E., \& Shuder, J. M. 1982, ApJS, 49, 149

Peng, C. Y. 2007, ApJ, 671, 1098

Peng, C. Y., Ho, L. C., Impey, C. D., \& Rix, H.-W. 2002, AJ, 124, 266

Peng, C. Y., Impey, C. D., Ho, L. C., Barton, E. J., \& Rix, H.-W. 2006a, ApJ, 640, 114

Peng, C. Y., Impey, C. D., Rix, H.-W., Kochanek, C. S., Keeton, C. R., Falco, E. E., Lehár, J., \& McLeod, B. A. 2006b, ApJ, 649, 616

Peterson, B. M., \& Wandel, A. 1999, ApJ, 521, L95

Peterson, B. M., et al. 2004, ApJ, 613, 682

Peterson, B. M., et al. 2005, ApJ, 632, 799

Pounds, K. A., Done, C., \& Osborne, J. P. 1995, MNRAS, 277, L5

Richards, G. T., Vanden Berk, D. E., Reichard, T. A., Hall, P. B., Schneider, D. P., SubbaRao, M., Thakar, A. R., \& York, D. G. 2002a, AJ, 124, 1

Richards, G. T., et al. 2002b, AJ, 123, 2945

Riechers, D. A., Walter, F., Carilli, C. L., Bertoldi, F., \& Momjian, E. 2008, ApJ, 686, L9

Riechers, D. A., Walter, F., Carilli, C. L., \& Lewis, G. F. 2009, ApJ, 690, 463

Robertson, B., Hernquist, L., Cox, T. J., Di Matteo, T., Hopkins, P. F., Martini, P., \& Springel, V. 2006, ApJ, 641, 90

Salviander, S., Shields, G. A., Gebhardt, K., \& Bonning, E. W. 2007, ApJ, 662 , 131

Sérsic, J. L. 1968, Atlas de galaxias australes (Cordoba, Argentina: Observatorio Astronomico, 1968)

Shen, J., Vanden Berk, D. E., Schneider, D. P., \& Hall, P. B. 2008a, AJ, 135, 928

Shen, Y., Greene, J. E., Strauss, M. A., Richards, G. T., \& Schneider, D. P. 2008b, ApJ, 680, 169

Shields, G. A., Gebhardt, K., Salviander, S., Wills, B. J., Xie, B., Brotherton, M. S., Yuan, J., \& Dietrich, M. 2003, ApJ, 583, 124

Shields, G. A., Menezes, K. L., Massart, C. A., \& Vanden Bout, P. 2006, ApJ, 641,683

Sigut, T. A. A., \& Pradhan, A. K. 2003, ApJS, 145, 15

Silk, J., \& Rees, M. J. 1998, A\&A, 331, L1

Somerville, R. S. 2009, MNRAS, 399, 1988

Storey, P. J., \& Hummer, D. G. 1995, MNRAS, 272, 41

Sulentic, J. W., Bachev, R., Marziani, P., Negrete, C. A., \& Dultzin, D. 2007, ApJ, 666,757

Thomas, J., Saglia, R. P., Bender, R., Thomas, D., Gebhardt, K., Magorrian, J., Corsini, E. M., \& Wegner, G. 2005, MNRAS, 360, 1355

Tremaine, S., et al. 2002, ApJ, 574, 740

Treu, T., Woo, J.-H., Malkan, M. A., \& Blandford, R. D. 2007, ApJ, 667, 117

Trujillo, I., et al. 2006, MNRAS, 373, L36

Vacca, W. D., Cushing, M. C., \& Rayner, J. T. 2003, PASP, 115, 389

van der Wel, A., Holden, B. P., Zirm, A. W., Franx, M., Rettura, A., Illingworth, G. D., \& Ford, H. C. 2008, ApJ, 688, 48

van Dokkum, P. G., et al. 2008, ApJ, 677, L5

Verner, E., Bruhweiler, F., Verner, D., Johansson, S., Kallman, T., \& Gull, T. 2004, ApJ, 611, 780

Vestergaard, M. 2002, ApJ, 571, 733

Vestergaard, M., Fan, X., Tremonti, C. A., Osmer, P. S., \& Richards, G. T. 2008, ApJ, 674, L1

Vestergaard, M., \& Peterson, B. M. 2006, ApJ, 641, 689

Vestergaard, M., \& Wilkes, B. J. 2001, ApJS, 134, 1

Walter, F., Carilli, C., Bertoldi, F., Menten, K., Cox, P., Lo, K. Y., Fan, X., \& Strauss, M. A. 2004, ApJ, 615, L17

Welsh, W. F., \& Horne, K. 1991, ApJ, 379, 586

Wilhite, B. C., Brunner, R. J., Schneider, D. P., \& Vanden Berk, D. E. 2007, ApJ, 669, 791

Wilson, J. C., et al. 2004, Proc. SPIE, 5492, 1295

Woo, J.-H., Treu, T., Malkan, M. A., \& Blandford, R. D. 2006, ApJ, 645, 900

Woo, J.-H., Treu, T., Malkan, M. A., \& Blandford, R. D. 2008, ApJ, 681, 925

York, D. G., et al. 2000, AJ, 120, 1579

Yu, Q., \& Tremaine, S. 2002, MNRAS, 335, 965 